\title{
Virtual Reality Avatar Therapy for People Hearing Voices
}

\section{Master Thesis}

Author(s):

Brander, Mischa

Publication date:

2020

Permanent link:

https://doi.org/10.3929/ethz-b-000445887

Rights / license:

In Copyright - Non-Commercial Use Permitted 


\title{
Virtual Reality Avatar Therapy for People Hearing Voices
}

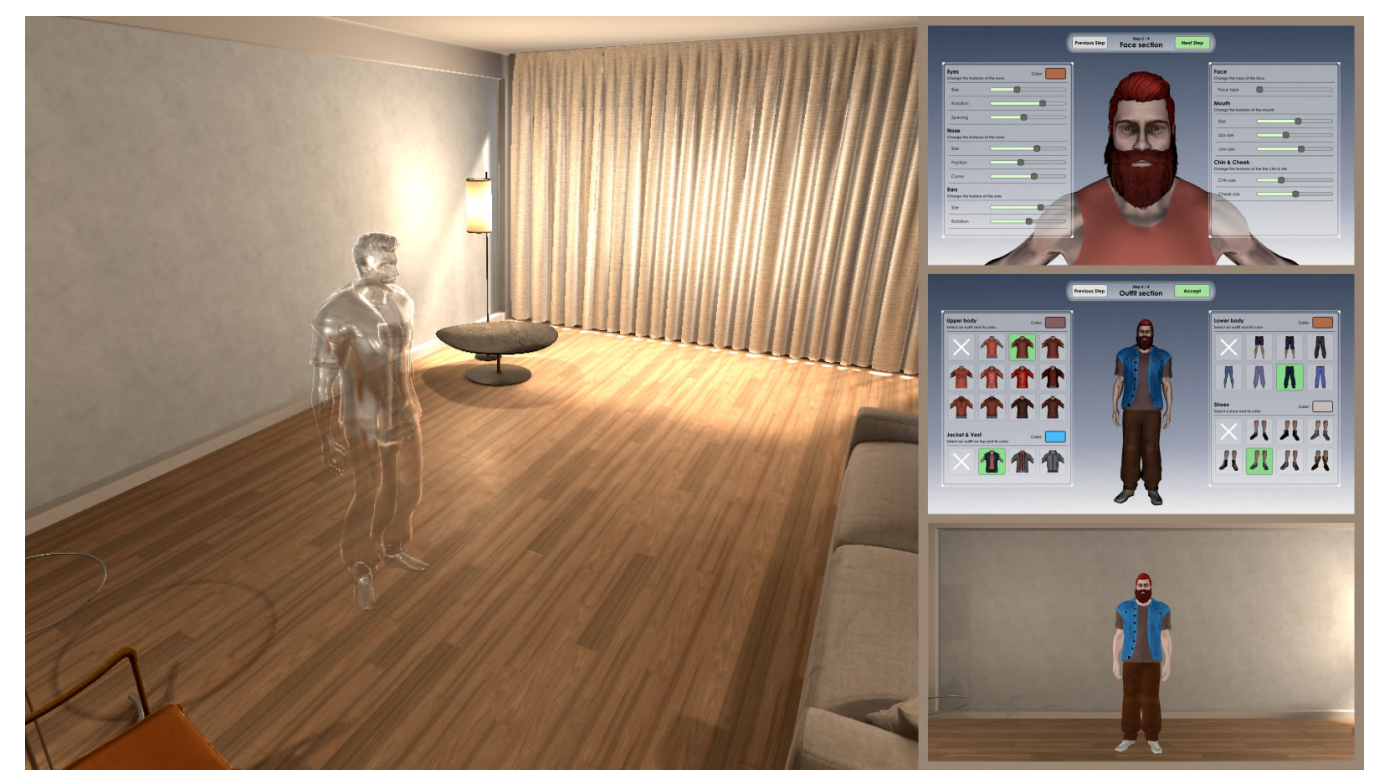

\author{
Mischa Brander
}

\author{
Master Thesis
}

October 2020

Dr. Stéphane Magnenat, Dr. Stephan T. Egger Prof. Dr. Robert W. Sumner

\section{EIH}

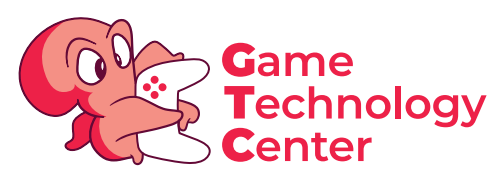





\section{Abstract}

Hearing voices is probably the worst kind of acoustic hallucinations, it can be experienced as severely disturbing and burdensome. It is influenced by diverse factors, one of them being the ability to interact and control the hallucinatory experience itself. In recent years, virtual reality has increasingly become a treatment option for those suffering from psychiatric disorder in general, and those with acoustic hallucination in particular. The goal of this thesis is the development of the technical part of a new therapeutic approach called Virtual Reality Avatar Therapy. It allows patients with acoustic hallucinations to design and customize a visual and auditory recreation of the avatar to which they attribute their acoustic hallucination. They can interact with their avatar in a virtual environment, while a therapist remotely controls the avatar and speaks through it using voice modification and lip synchronization. The created system will be used at the Psychiatric University Hospital of Zurich in clinical and research practice; including a study to investigate the efficacy and feasibility of Virtual Reality Avatar Therapy of people hearing voices. The System Usability Scale (SUS) is used to evaluate the functionality and operability of the system by the medical and therapeutic staff actually performing the therapy. The SUS yields a global score of 78.85 out of 100; showing a good benchmark for the usability and acceptance of the system, and all components to support the clinical trial are provided. 



\section{Acknowledgements}

Throughout my master thesis I have received a great support and assistance. I would like to thank the following people for their great help.

First, I would like to thank my supervisors, Dr. Stéphane Magnenat and Dr. Stephan T. Egger. They supported me throughout my thesis with their excellent expertise and weekly feedbacks. Their doors were always open, and they helped me find the right way to finish my project.

I would also like to thank Prof. Dr. Robert W. Sumner to oversee my thesis and make it possible to write it at the Game Technology Center. It was a pleasure to be a part of the GTC.

Further, I would like to acknowledge the team at the Psychiatric University Hospital of Zurich in Rheinau for their regular testing and helpful feedback. Also, the intensive testing and filling out of the System Usability Scale questionnaire at the end of the project would not have been possible without them. Thank you.

Finally, I would like to thank the team at the Game Technology Center for the inspiring and pleasant weekly meetings, their feedback and support, and the friendly collaboration. 



\section{Contents}

List of Figures vii

List of Tables $\quad$ ix

1. Introduction 1

2. Related Work 3

3. Project setup 7

3.1. Requirements analysis . . . . . . . . . . . . . . 7

3.1.1. Hardware requirements . . . . . . . . . . . . . . . . . . . 7

3.1.2. Application requirements . . . . . . . . . . . . . . . . . . . . . 11

3.2. System design . . . . . . . . . . . . . . . . . . . . . . . 12

4. Implementation $\quad 15$

4.1. Network protocol . . . . . . . . . . . . . . . . . . 16

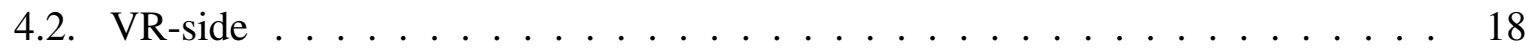

4.2.1. Avatar customization . . . . . . . . . . . . . . . . . . . . . . 19

4.2.2. VR scene ............................ 22

4.3. Therapist-side . . . . . . . . . . . . . . . . . . . 24

4.3.1. Voice modification . . . . . . . . . . . . . . . . . . . 27

4.3.2. Gesture control . . . . . . . . . . . . . . . 28

5. Evaluation 31

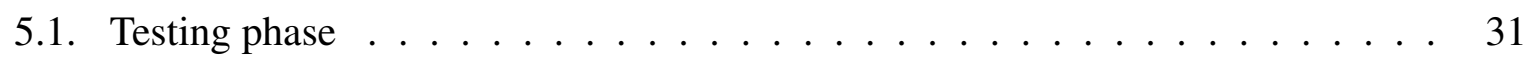

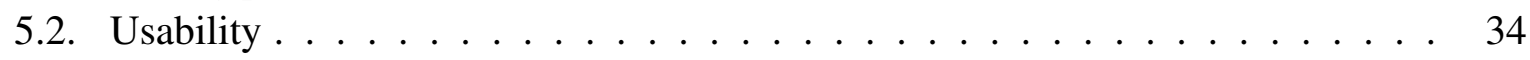


6. Conclusion 39

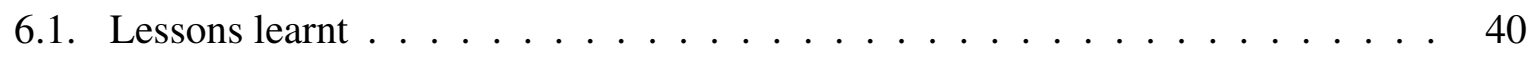

6.2. Future Work . . . . . . . . . . . . . . . . . . 40

$\begin{array}{ll}\text { A. Appendix } & 43\end{array}$

$\begin{array}{ll}\text { Bibliography } & 47\end{array}$ 


\section{List of Figures}

3.1. HP Reverb headset with motion controllers [3]. . . . . . . . . . . . . . 10

3.2. Medion Erazer X87092 with a glass side window [4] . . . . . . . . . . . . 10

3.3. System overview of the hardware. . . . . . . . . . . . . . . . . . . . 12

3.4. Block diagram of the application. . . . . . . . . . . . . . . 13

4.1. Environments for implementing the applications. . . . . . . . . . . 15

4.2. Block diagram of the network protocol. . . . . . . . . . . . . . . 17

4.3. Start scene showing available therapies. . . . . . . . . . . . . . . 18

4.4. Collection of different hairstyles for UMA. . . . . . . . . . . . . . . . . 20

4.5. Collection of different outfits for UMA. . . . . . . . . . . . . . . . . 20

4.6. The four steps of the avatar customization. . . . . . . . . . . . . . . 22

4.7. View of the therapy room within Unity. . . . . . . . . . . . . . . 23

4.8. Start scene asking for the IP-address. . . . . . . . . . . . . . . . . . . . . 24

4.9. Blank therapy scene showing the control panel. . . . . . . . . . . . 25

4.10. Therapy scene from different camera views. . . . . . . . . . . . . . 26

4.11. Roland VT-4 Voice Transformer [5] . . . . . . . . . . . . . . . . . . . . 27

4.12. Different mood states of the avatar. . . . . . . . . . . . . . . . . 29

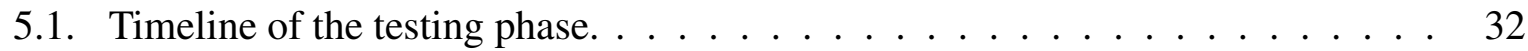

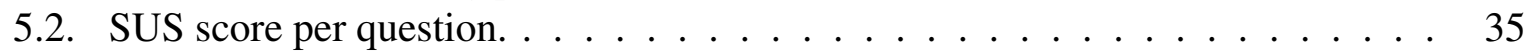

5.3. A histogram of individual SUS scores. . . . . . . . . . . . . . . 36

5.4. Total SUS score for both applications. . . . . . . . . . . . . . . . . . . . . . . . . .

5.5. Comparison of mean SUS scores [23] . . . . . . . . . . . . . . . 37

5.6. SUS score per profession - VR application. . . . . . . . . . . . . . . 37

5.7. SUS score per profession - Therapist's application. . . . . . . . . . . . 38 



\section{List of Tables}

3.1. Hardware requirements for VRAT $\ldots \ldots \ldots \ldots \ldots \ldots \ldots$

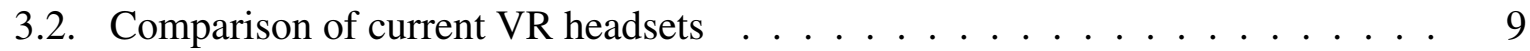

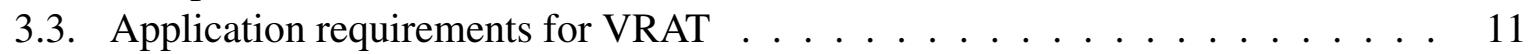

4.1. Overview of the different data messages $\ldots \ldots \ldots \ldots \ldots$

5.1. Questions for testing the avatar customization . . . . . . . . . . 32

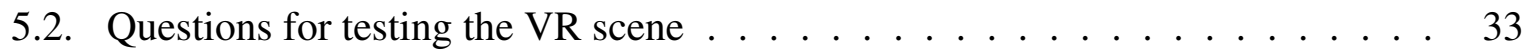

5.3. Questions for testing the therapist-side . . . . . . . . . . . 33

5.4. Questions of the System Usability Scale . . . . . . . . . . . . . . . . 34

A.1. Comparison of current VR headsets - Tethered . . . . . . . . . . . . 43

A.2. Comparison of current VR headsets - Standalone . . . . . . . . . . . . 44

A.3. Comparison of current VR headsets - Cardboard . . . . . . . . . . . 44

A.4. PC requirements for tethered VR headsets . . . . . . . . . . . . . 44

A.5. Data set of the SUS questionnaire - Part $1 \ldots \ldots \ldots \ldots$

A.6. Data set of the SUS questionnaire - Part $2 \ldots \ldots \ldots$ 



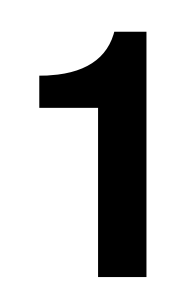

\section{Introduction}

Hearing voices is a form of acoustic hallucinations, where those affected perceive a voice; sometimes even with a commentating character. This experience can become severely disrupting and burdensome, lastly depending on diverse factors like the content itself, the possibility of interaction and the ability of the individual to influence and control the hallucinatory experience itself. In the last few years, a new form of therapy appeared, the so-called Avatar Therapy. It enables for those affected an interaction with the voice in a new way by adding a visual layer. In this therapy, a patient designs a visual and auditory avatar, resembling the person believed to be the source of the voice they hear; in the case they hear several voices they have to choose the most bothersome one. During a therapy session, the patient can interact with the created avatar, which is controlled by a therapist. This interaction should help the patient to get control over the interaction with the avatar and transfer this experience to gain control over the acoustic hallucination. To enhance the immersion of the avatar therapy, Virtual Reality (VR) can be used, whereby the patient interacts with the avatar in a virtual environment.

VR simulates an immersive environment using computer technology. The user immerses fully into the virtual world by wearing a Head-Mounted Display (HMD). The HMD provides a stereoscopic display, which shows for each eye a different image leading to a 3D effect; thus creating the immersive environment. The headset and a complementary set of tracking and motion controllers, as well as a sound system allow the user to interact with the designed virtual world. To date there exist three types of HMD: 1 . Connected to a computer, called tethered; 2 . standalone devices; and 3. cardboards used for smartphones. Each of these devices has a processing unit, which calculates the 3D world and constantly renders the scenery. VR applications are mostly used in the gaming industry, but are also used for mental health, sport or education.

The implementation of the Avatar Therapy through VR will be used in clinical and research practice, including a study in the Psychiatric University Hospital of Zurich (PUK). The goal of the study is to analyse the feasibility and efficacy of a Virtual Reality Avatar Therapy (VRAT) approach in patients with acoustic hallucinations, with the aim to achieve an improvement in 


\section{Introduction}

general symptomatology, especially the hallucinatory experience itself, as well as to gain quality of life. The study has a single-blind, randomised, controlled trial in a cross-over design, where participants will be scheduled to either nine sessions of VRAT or nine sessions of an Assertiveness Training Program [25]; switching treatment groups after three weeks. In the VRAT, patients create the appearance and voice of the avatar, with the help of their therapist. Over the rest of the therapy, the patient interacts with the avatar controlled by the therapist in dialogue, gaining control over the situation and influencing the avatar and the content of the discourse. The interaction should help the patient to get more self-confident and to get more control over the acoustic hallucinations itself.

The goal of this master thesis is to develop a system, which provides the technical platform to perform the VRAT intervention as a "Serious Game". This includes researching other similar experiences and relevant studies; choosing and setting up the required hardware; implementing the according software application; and finally testing it together with the therapeutic team at the clinic site. The system itself provides a VR environment, an avatar customization tool, a voice chat to let the therapist remotely talk to the patient, a lip synchronization tool to increase the immersion, and a set of animations to give the avatar natural movements. 


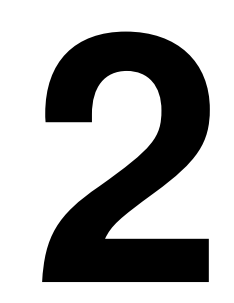

\section{Related Work}

The last few years saw an increase in the use of VR applications for treatment of mental disorders, like anxiety and phobia, Post-Traumatic Stress Disorder (PTSD) and schizophrenia. Mostly VR served as an alternative to exposure therapy, where the patients get confronted with the source of their despair. In the past, real world objects and situations were used for therapy, but with VR new technical opportunities and possibilities arise. VR can create a virtual detailed representation of the source of despair and simulate a suitable exposure environment and gain control of the situation. Like standing on the top of a high building for those with fear of heights or sitting in a plane for those with fear of flying [28].

VR allows to simulate situations, which are difficult to achieve in the real world, and can reduce the effort of creating such a situation. Furthermore, this gives full control of the exposure intensity and duration; as well as safe and constantly available exit strategy. Recently VR was also used in the treatment of people with a schizophrenia; enabling them to interact with acoustic hallucinations, or gaining confidence in certain social situations experienced as bothersome. The main element of the treatment is a so-called Avatar Therapy [26], where patients have the possibility to interact with a visual and auditory recreation of their acoustic hallucination through an avatar. The avatar is a virtually-created character by the patient itself, which represents the entity behind the voice. A therapist takes over the control of the avatar and speaks through it using voice modification; thus enabling a dialogue with the patient. The therapist leads this conversational interaction; as a therapeutic intervention addressing situations where the acoustic hallucinations cause distress or suffering. This Avatar Therapy can be combined with VR, to increase the immersion for the patient and creating a new kind of interaction.

Currently, there exist some related studies in this topic, showing a first approach of using avatars for people hearing voices. In a first study, the Avatar Therapy is tested without VR. Between November 2013 and January 2016 a study for an Avatar Therapy for auditory verbal hallucinations in people with schizophrenia [26] was conducted in London. 150 eligible participants with acoustic hallucinations were tested. The authors studied the effect of an avatar therapy on 
the severity of acoustic hallucinations. Each participant was randomly assigned to either the avatar therapy or the control group, receiving supportive counselling. After the therapy, two follow-up examinations were held, 12 weeks and 24 weeks later. From the technical perspective, the authors chose an avatar recreation on a $2 \mathrm{D}$ screen. The participants interact with the avatar directly on the monitor for up to 15 minutes, whereby the therapist controlled the avatar. The authors provided a control panel for the therapist in another room, which allows speaking to the patient with either a normal voice or a manipulated voice, representing the voice of the avatar. The results show a reduction in the severity of auditory hallucinations and that it is more effective than the control therapy. Overall, the study shows a first evidence for the benefit of using an Avatar Therapy for people hearing voices.

In another study from 2018 [27], a team from Montreal tested a VR-assisted therapy with 19 patients and compared it to an anti-psychotic treatment, with regular therapeutic meetings with clinicians. The VR-assisted therapy consists of 7 therapy sessions, one for creating an avatar and six for the actual therapeutic interaction. The patients create an avatar and its voice, believed to be the source of the malevolent voice, which they hear. To create the avatar, the authors used the Unity 3D game engine [21] and the Morph3D Character System. The voice is simulated in real-time with a voice modification device and the lip synchronization is performed via a Unity extension called Simple Automated Lip Sync Approximation (SALSA) [12]. The patient puts on a Samsung GearVR HMD to experience the VR avatar scene, which runs on a Samsung Galaxy S6 smartphone. In the virtual world, the patient is in a dark room and sees the avatar from a first-person perspective. The therapist controls the avatar and creates a dialogue between the patient and the avatar with provided sentences. The results of the study show also a significant improvement in the quality of life and reduction of the related distress of the patients with the new therapy form. The authors state, that a VR assisted therapy is a highly novel and promising intervention for refractory auditory verbal hallucinations in schizophrenia.

Both studies present good results, whereby a lot of the participants notice a decrease in hearing the voices. The results are therefore promising, but they are not conclusive and further research is considered necessary. From a technical point of view, the immersion in both studies could be much higher for the patients. The first study tests the Avatar Therapy without VR. The avatar is represented only as a floating face on a black background. The interaction for the user is limited to the interaction with a $2 \mathrm{D}$ monitor, and the movement of the avatar is restricted to the lip synchronization. The avatar does not represent a full 3D character, since the body is missing. Therefore, the user cannot completely customize the avatar. In the second study an initial approach of using VR is tested. This time, the patients can see the avatar through a HMD. But the VR scene only consists of the face of the avatar and is otherwise completely black. The patients sit directly in front of the face, and the scenery lowers their immersion. Also, the computational power of the application is limited, since a cardboard system with a Samsung Galaxy S6 smartphone is provided. It allows an easy setup but limits the use of VR.

In this project, the idea is to increase the level of interaction and to increase the immersion for the patient. For this reason, a new and improved VR approach is designed and developed. The avatar is represented as a full 3D character, with a complete body and an outfit. Therefore, additional features to customize the avatar are provided and the patients can create a complete avatar on their own. The customization process of the therapy is seen as a very important step. A Team from the USA evaluated in a study in 2014 the effect of avatar-based customization on players identification [29]. 
The authors tested the player's identification with their characters, and the empathy towards them. The players were either playing an online game with a self-customized avatar or a predefined one. The authors showed a positive impact for the player's identification with their avatars in the group of the self-customized avatars. This identification increased the overall feelings and experience of the players. The analysed effect of the study could also be important for the VRAT, to increase the efficiency of the interaction between the patients and their avatars. The increased empathy towards the created avatar could help to get a better control of the avatar and the voice itself. Further, the VR experience is enhanced in this project with a more powerful setup. A computer connected headset increases the image quality and provides a higher immersion for the user. Also, the black scene is replaced with a representation of a virtual therapy room. The patient can also stand in the room, look $360^{\circ}$ around, and move slightly. The immersion is further increased with the possibility of the avatar to change its mood, controlled by the therapist. This gives the avatar more personality and the option to show feelings. 



\section{3}

\section{Project setup}

\subsection{Requirements analysis}

An important step of the project is the requirements analysis to clarify in advance which components are needed to achieve the goal of creating a fully working VRAT. The technical structure of the VRAT is conceived as follows; with a session room where the patients get immersed in the VR; a separate room from where the therapist controls the voice of the avatar and the interaction; as well as a way to transmit the spoken language between both of these rooms.

\subsubsection{Hardware requirements}

The hardware devices need to support the use of VR and the creation of a virtual avatar. A VR HMD together with a high-end computer form the basis for the VR system. For providing a real-time manipulation of the voice of the therapist, a voice transformer and a headset is used. Further, since the network for the therapy should be independent of the existing network in the clinic, an own one is used to establish a connection between the two rooms. Finally, a monitor, a mouse, a keyboard, and speakers assist the creation of the avatar and its voice. Table 3.1 lists the hardware requirements.

Currently, there exist a wide range of different producers and models of VR headsets. A comparison of them helps to find an appropriate one for the therapy. The VR headset is used during the VRAT to immerse the patient. This leads to some requirements. First, the application represents a virtual simulation of a therapy and should provide a high immersion with a good image quality. Further, there are multiple users of the headset and the therapy room could change over time, which leads to the requirement of an easy setup. 
Table 3.1.: Hardware requirements for VRAT.

\begin{tabular}{|c|c|}
\hline Requirement & Description \\
\hline VR headset & Let patients immerse into the VR environment \\
\hline Computer & Renders the VR scene, provides avatar customization \\
\hline Monitor & Shows the running application (connected to computer) \\
\hline Mouse \& Keyboard & Listens to user input (connected to the computer) \\
\hline Speakers & Outputs the transformed voice during the creation step \\
\hline Laptop & Allows therapist to control the therapy \\
\hline Voice transformer & Transforms therapist's voice in real time \\
\hline Headset & Allows therapist to chat with the patient \\
\hline WLAN router & Connects therapist's laptop with the computer \\
\hline
\end{tabular}

The relevant elements for the image quality of a headset are the display type, the Field of View (FOV), the resolution and the refresh rate. There exists different types of the display, but mostly there are Liquid Crystal Displays (LCD) or Organic Light Emitting Diodes (OLED) used [20]. The possible extension to use an active matrix for the OLED exists, called Active Matrix OLED (AMOLED). LCD are the cheapest ones, having difficulty to display true black and having a worse color accuracy. In this list, the FOV defines the horizontal angle span that the user can see at the same time. A larger view field increases the immersion but needs more computing power to render the scene. Another important factor is the resolution of the display, given as pixel width and height for both eyes. A higher resolution leads to a clearer, more detailed image and reduces the so called Screen-Door effect, where the user can see some visible gaps between pixels [13]. Finally, the refresh rate of the device must be considered. It defines the number of new images that the headset can display in one second. A low refresh rate produces flickering and can induce disorientation or other negative effects.

Important for an easy setup and a high usability of the VR headset are the tracking system and additional features, like the integrated sound system. There exists mostly two different types of tracking systems: Inside-out tracking or outside-in tracking [6], whereby inside-out tracking is more often used in recent years. For inside-out tracking, the HMD needs some attached cameras to track itself in the room. In the outside-in system, cameras are placed in the room and usually track a set of markers attached to the HMD. The tracking does not depend on the room content or visual features, and is therefore more robust. It provides a more accurate tracking of the headset and controllers, but comes with a setup overhead.

Besides the display and the tracking system, the integration of headphones and a microphone for the communication is also relevant. The platform for the VR headset is mostly relevant for the developing process and should be supported by Windows 10. The comfort of the HMD is also highly relevant since it can influence the final acceptability of the participants. And finally, the prices of the devices are compared to definitively take a decision. The prices are shown in the list in the Appendix. 
In Table 3.2, some relevant and current available VR headsets are compared. Since the final used headset is a tethered one (connected to a computer), only tethered headsets are listed, to compare them better. A list of the complete comparison is provided in the Appendix.

Table 3.2.: Comparison of current VR headsets.

\begin{tabular}{l|l|l|l|l|l|l|l}
\hline Name & Producer & Display & FOV & Resolution & Rate & Tracking & Platform \\
\hline Acer AH101 & Acer & LCD & $95^{\circ}$ & $2880 \times 1440$ & $90 \mathrm{~Hz}$ & Inside-out & WMR \\
Fove & Vove Inc. & OLED & $100^{\circ}$ & $2560 \times 1440$ & $70 \mathrm{~Hz}$ & Outside-in & SteamVR \\
HP Reverb & HP Inc. & LCD & $114^{\circ}$ & $4320 \times 2160$ & $90 \mathrm{~Hz}$ & Inside-out & WMR \\
HTC Vive & HTC & AMOLED & $110^{\circ}$ & $2160 \times 1200$ & $90 \mathrm{~Hz}$ & Outside-in & SteamVR \\
HTC Vive Cosmos & HTC & LCD & $110^{\circ}$ & $2880 \times 1700$ & $90 \mathrm{~Hz}$ & Inside-out & SteamVR \\
HTC Vive Pro & HTC & AMOLED & $110^{\circ}$ & $2880 \times 1600$ & $90 \mathrm{~Hz}$ & Outside-in & SteamVR \\
Lenovo Explorer & Lenovo & LCD & $110^{\circ}$ & $2880 \times 1440$ & $90 \mathrm{~Hz}$ & Inside-out & WMR \\
Oculus Rift & Oculus & OLED & $110^{\circ}$ & $2160 \times 1200$ & $90 \mathrm{~Hz}$ & Outside-in & Oculus \\
Oculus Rift S & Oculus & LCD & $115^{\circ}$ & $2560 \times 1440$ & $80 \mathrm{~Hz}$ & Inside-out & Oculus \\
Pimax 5K + & Pimax & LCD & $170^{\circ}$ & $5120 \times 1440$ & $144 \mathrm{~Hz}$ & Outside-in & SteamVR \\
Pimax 8K & Pimax & LCD & $170^{\circ}$ & $7680 \times 2160$ & $80 \mathrm{~Hz}$ & Outside-in & SteamVR \\
Pimax 8K X & Pimax & LCD & $200^{\circ}$ & $7680 \times 2160$ & $80 \mathrm{~Hz}$ & Outside-in & SteamVR \\
Playstation VR & Sony & OLED & $100^{\circ}$ & $1920 \times 1080$ & $120 \mathrm{~Hz}$ & Outside-in & PS4 \\
Samsung Odyssey+ & Samsung & AMOLED & $110^{\circ}$ & $3200 \times 1440$ & $90 \mathrm{~Hz}$ & Inside-out & WMR \\
StarVR One & StarVR & AMOLED & $210^{\circ}$ & $3660 \times 1464$ & $90 \mathrm{~Hz}$ & Outside-in & SteamVR \\
Valve Index & Valve & LCD & $130^{\circ}$ & $2880 \times 1600$ & $144 \mathrm{~Hz}$ & Outside-in & SteamVR \\
Varjo VR-1 & Varjo & AMOLED & $87^{\circ}$ & $1920 \times 1080$ & $90 \mathrm{~Hz}$ & Outside-in & SteamVR \\
XTAL & Vrgineers & OLED & $170^{\circ}$ & $5120 \times 1440$ & $70 \mathrm{~Hz}$ & Outside-in & SteamVR
\end{tabular}

As a result of the comparison, a HP Reverb headset [2] from HP Inc. meets the given requirements. It has a $4320 \times 2160$ dual LCD with a refresh rate of $90 \mathrm{~Hz}$ and a FOV of $114^{\circ}$, which altogether provides a good image quality for the therapy. The headset is recommended for simulations, has integrated headphones/microphone and comes with an inside-out tracking system with two front facing cameras allowing 6 Degrees of Freedom (DOF) motion tracking. Since the patient is mostly standing at one position and no controllers are involved during the therapy, the easy setup of the inside-out tracking is preferred over the more accurate one from the outside-in tracking system. The HP Reverb is a Windows Mixed Reality (WMR) headset and comes with the Mixed Reality Portal [10] application for Windows 10. 


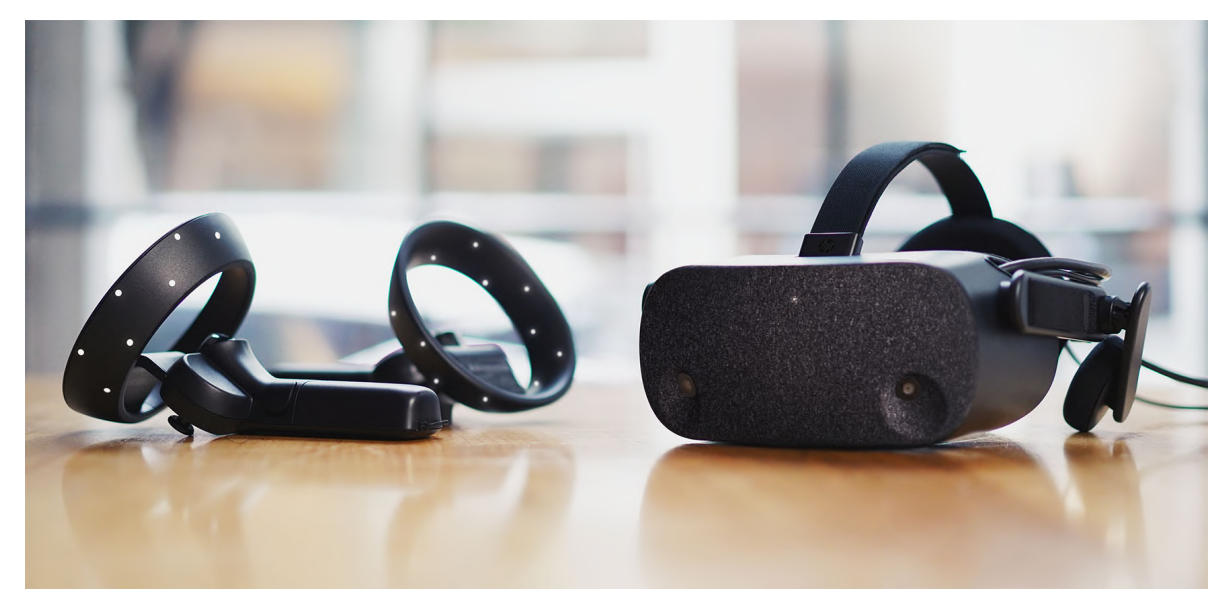

Figure 3.1.: HP Reverb headset with motion controllers [3].

The HP Reverb headset has to be connected to a computer and comes with a series of computational requirements; which are determinant at the moment of choosing a computer. The producer recommends for a minimum usage a Nvidia GeForce GTX 1080 graphic card or an AMD Radeon Pro WX 8200, an Intel Core i7 processor, 16 GB of RAM, a display port 1.3, and a USB port 3.0. For the operating system, Windows 10 (Version 1809 or later) is needed [14]. To get an excellent image quality and to use the computer for possible future therapies, a suitable one is purchased. A Medion Erazer X87092 [7] from Medion. It has to render the VR scene, show the avatar customization and support the development and debugging process. It comes with a Nvidia GeForce RTX 2070 Super graphic card, an Intel Core i7-9700 processor, 16 GB of DDR4 RAM, 512 GB SSD storage, the needed ports, and Windows 10 Home is installed.

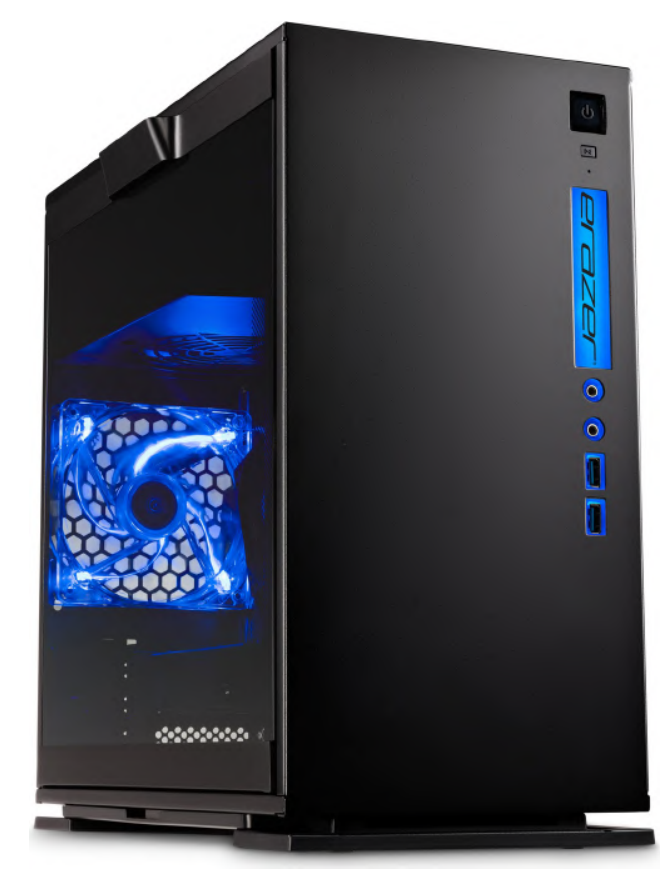

Figure 3.2.: Medion Erazer X87092 with a glass side window [4]. 


\subsubsection{Application requirements}

The second part of the system is the application part. Two applications are implemented. One provides the VR experience and the avatar customization process, the other provides a control and monitor application for the therapist to get access to the therapy. Both applications need a GUI for user interaction, a 3D environment and a character system. More requirements for the applications are listed in Table 3.3.

Table 3.3.: Application requirements for VRAT.

\begin{tabular}{|c|c|}
\hline Requirement & Description \\
\hline Game engine & Offers a framework to develop the application \\
\hline $3 \mathrm{D}$ character system & Create a 3D avatar representation \\
\hline Lip synchronization & Move the lips of the avatar while talking \\
\hline Animations & Animate the avatar during a therapy session \\
\hline Network protocol & Allow TCP / UDP connection between server-client \\
\hline Voice chat & Transmit microphone input to audio source \\
\hline 3D models & Build a virtual therapy room \\
\hline Lighting & Illuminate the scene \\
\hline VR camera system & Setup camera to view the scene in VR \\
\hline VR platform & Start VR headset on the computer \\
\hline GUI & Provide an interface for the users \\
\hline
\end{tabular}

The 3D character system, the lip synchronization and the animations are used to build the main part of the therapy, the virtual avatar. The avatar is a 3D humanoid, standing in front of the patient with the ability to move its lip while talking and move parts of its body to make gestures. The main purpose of the network protocol is the support of a simple voice chat, which allows the therapist to talk with the patient remotely. Besides, a familiar looking virtual environment where the interaction with the avatar takes place is required. This is achieved by representing a virtual therapy room, with a 3D model of the room itself, some models for the furniture, and some lights. To finally use the application with a virtual headset, the matching VR platform has to be installed on the computer and the therapy scene must contain a camera, representing the view from a first-person perspective.

The Unity game engine, developed by Unity Technologies is a free available platform for game development, and over 60 percent of the current Augmented Reality (AR)/VR content is created with Unity [24]. It provides a 3D editor, a scripting API written in C\#, and allows to bring all components together supporting 3D-graphic, socket communication, and VR. Further, for implementing external plugins or tools, the Unity Asset Store [22] offers a big collection of assets, which can be easily integrated into an existing project. Unity also provides an own 
UI system, a tool for lighting, and a tool to create animations. The implementation of the applications is further discussed in Chapter 4.

\subsection{System design}

This project consists of two separated sides, one for the VR experience and the avatar itself, the other for the therapist as a control center. The hardware is directly installed in the therapy room. Table 3.1 lists the components and Figure 3.3 shows an overview of the hardware setup.

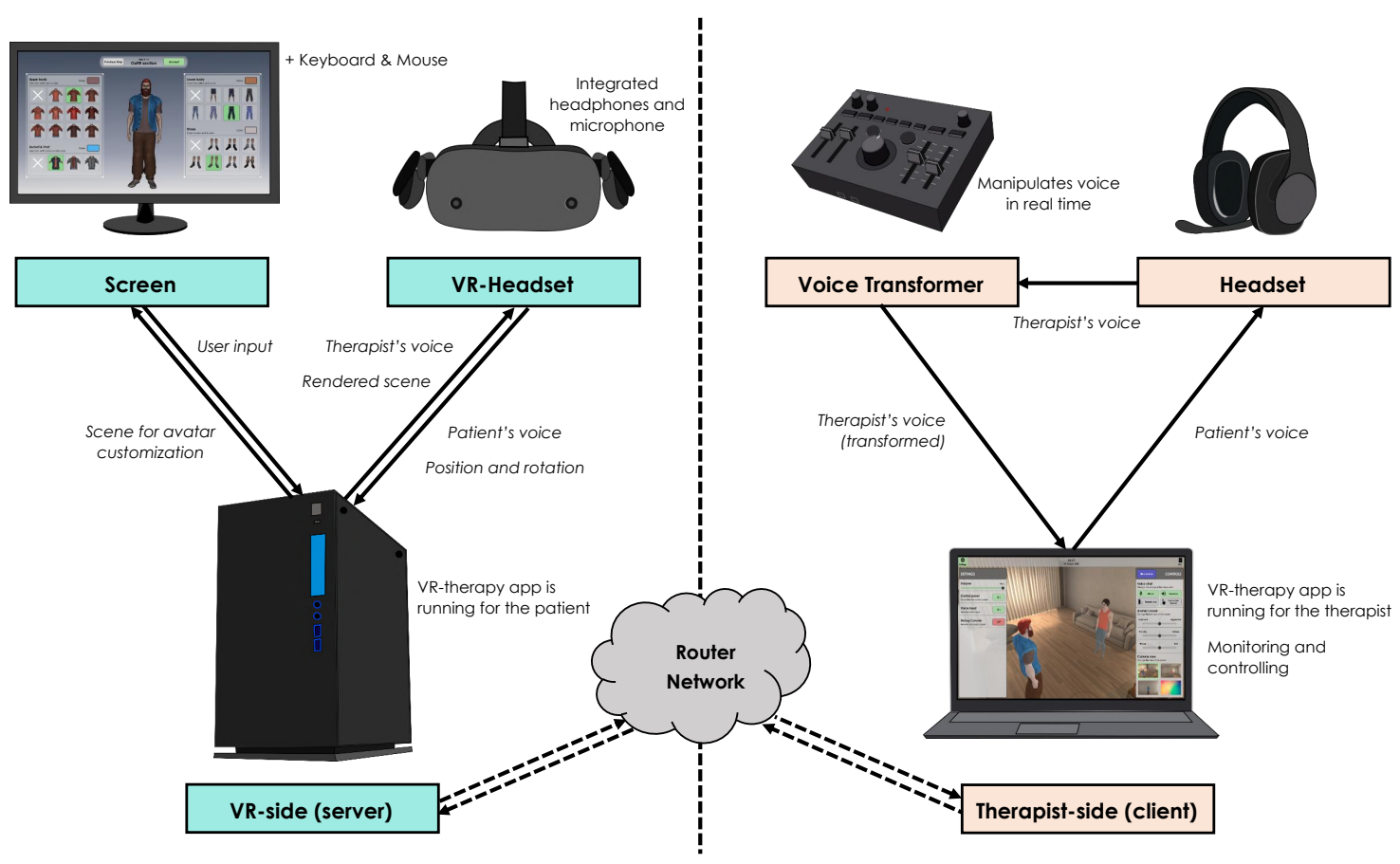

Figure 3.3.: System overview of the hardware.

On the VR-side, the Medion Erazer X87092 runs the Unity application for creating an avatar and renders the VR scene for the HP Reverb headset. The integrated microphone records the voice, and the headset itself tracks the position and rotation of the patient. Both information are sent to the server. The monitor, the keyboard, and the mouse are connected to the computer. Together with the speakers, they support the creation step of the avatar and its voice.

The second part is the therapist-side, where the second application runs to get access to the virtual therapy on a laptop. The therapist can join, monitor and control the therapy session from there; therefore, it is called control center. To remotely speak with the patient, a PC headset with a microphone and headphones is provided. The voice of the therapist is routed through a voice transformer placed between the microphone and the laptop. The transformed voice is then transferred over USB to the laptop and from there to the avatar. The headphones also allow the therapist to listen to the patient. 
To connect the VR-side with the therapist-side a local network is implemented. Therefore, a Fritz!Box WLAN router is used and installed in the therapy room.

To get a better overview of the implemented applications, Figure 3.4 shows a block diagram of the applications and presents an overview of the different created scenes.

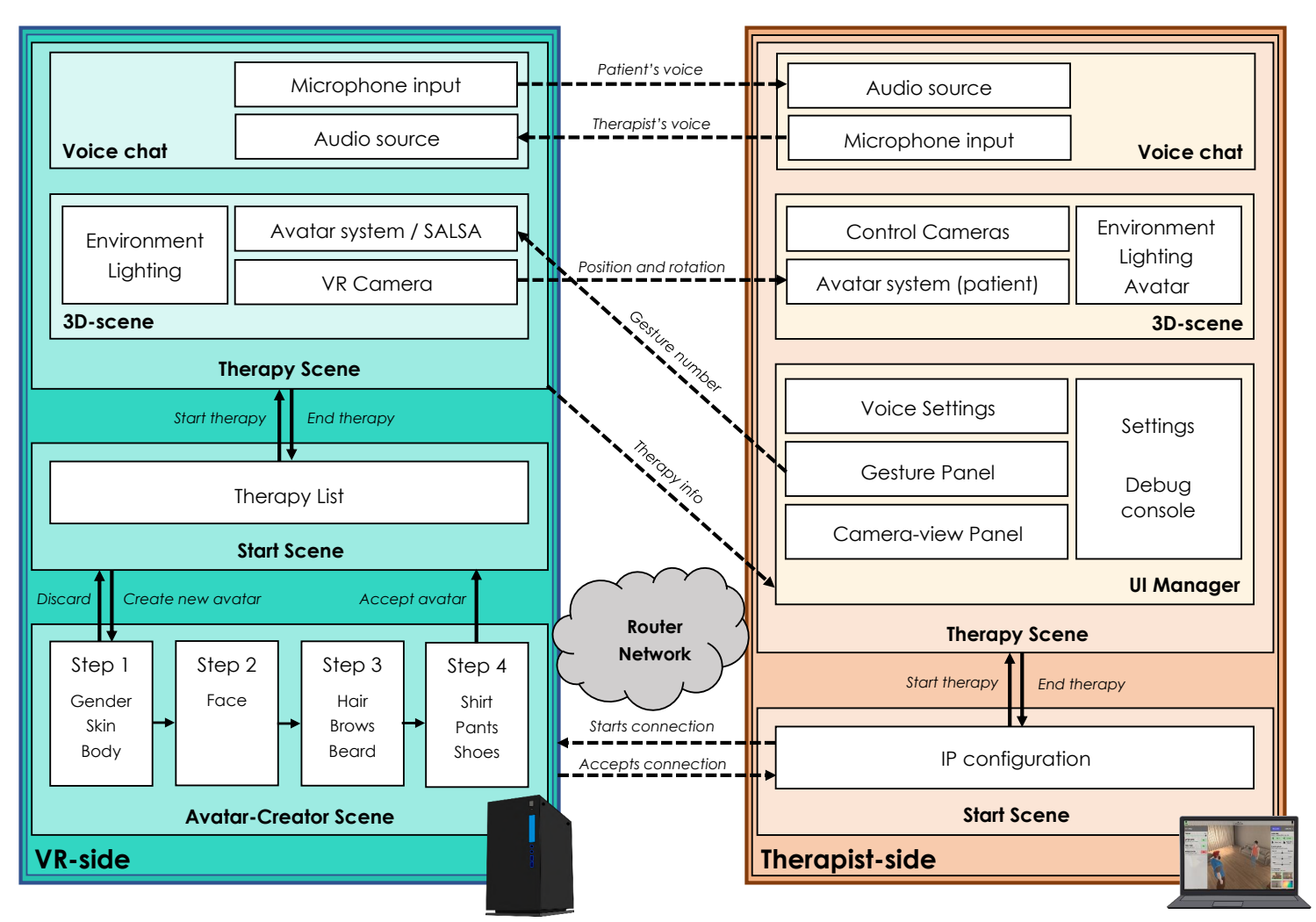

Figure 3.4.: Block diagram of the application.

The VR-side consists of three different scenes: The start scene, the avatar customization scene for creating an avatar, and a main scene. The main scene represents a virtual therapy room, where the patient can interact with the avatar. The avatar customization scene provides a tool for creating a 3D avatar. For the 3D character an open source avatar creation framework called Unity Multipurpose Avatar (UMA) [16] is used. It provides the code and some assets to create an own avatar customizer. Additionally, there exists enough content in the Unity Asset Store to expand the visual appearance of the avatar. Also lip synchronization is possible as Unity easily supports the integration of SALSA [12]. SALSA itself provides a high-quality lip synchronization for $2 \mathrm{D}$ and $3 \mathrm{D}$ characters in real-time and adds some animations for the eyes, eyelid and head, to let the avatar look around.

On the therapist-side are two scenes: A start scene and the therapy scene for the therapist. The therapy scene consists of a copy of the 3D scene of the therapy room, the avatar, as well as a default character, representing the patient. This allows the therapist to monitor the therapy. Further, a UI is provided to give the therapist control over the therapy, more precise, to control 


\section{Project setup}

the mood of the avatar, the camera view and the settings for a voice chat. A custom-designed voice chat system is implemented on both sides and allows a conversation. Both sides are connected over a TCP socket connection, where the VR-side assumes the server part, and the therapist-side the role of a client. In addition to the voice chat data, also the position and rotation of the VR headset, the data of the avatar and some control actions from the therapist are sent over the network. The rationale for implementing a home-made voice chat is to keep full control of the audio data. There is no need for an external server and the voice data never leave the internal network. 


\section{4}

\section{Implementation}

The implemented system consists of two separated applications running each on a different device. Both applications are created with Unity 2019.3.7 and the associated scripts are written in C\# with Microsoft Visual Studio 2017 [8], an Integrated development environment (IDE) from Microsoft. To develop and test the applications, Unity provides a 3D editor, where socalled Game Objects, the fundamental objects of Unity, can be placed and arranged into one or multiple scenes. Examples for a game object are 3D objects, UI elements, lights or cameras. Each of them can contain different components, which defines the logic and purpose of the object, like a transform component defining the position, rotation and scale of the object, an image component, an animator or a user created code script. The different game objects can be easily managed in a tree and the different components can be edited with the inspector tool. Unity provides many more additional tools, from which some are used for this project and are later described.

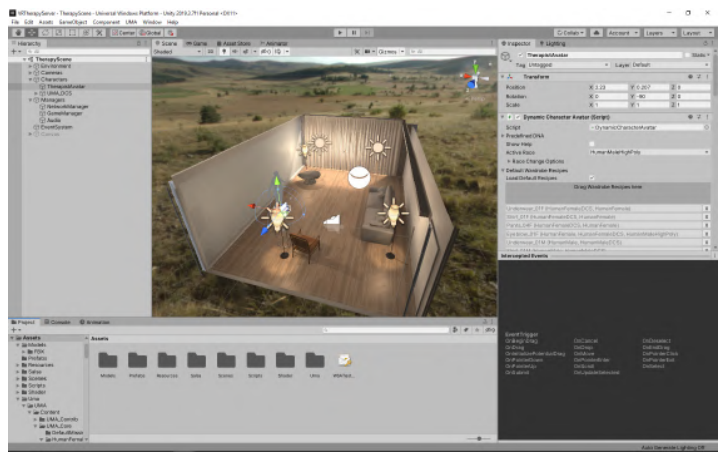

(a) Unity Editor

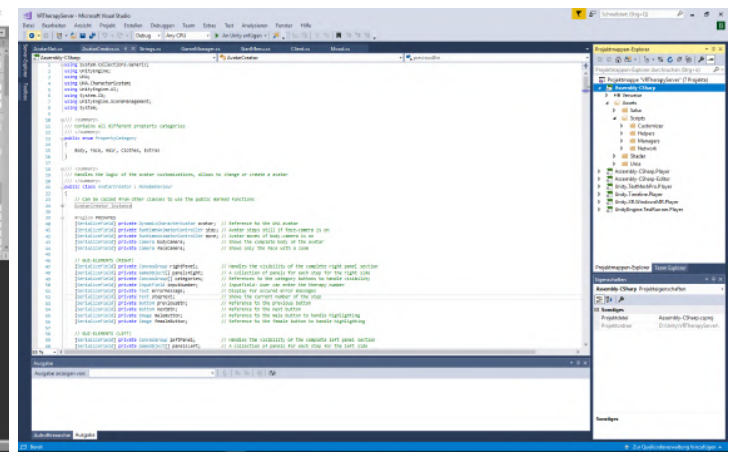

(b) Visual Studio

Figure 4.1.: Environments for implementing the applications. 


\section{Implementation}

In a first step, the VR-side is created, which provides the avatar customization tool and the VR scene, showing a virtual therapy room and the avatar. Next, in a second step, the therapist-side, the so-called control center is implemented. It consists of a copy of the therapy room to monitor it and a control panel to give the therapist control over the therapy and the avatar. The third step of the implementation is the creation of a custom-designed network protocol to connect both sides. The network is mostly used for a voice chat, which allows the therapist to speak with the patient through the avatar using voice modification.

\subsection{Network protocol}

To send data from the therapist-side to the VR-side and vice versa, a client-server connection is built. It supports the Transmission Control Protocol (TCP) and the User Datagram Protocol (UDP) and is implemented directly in Unity itself using the System.Net and System.Net.Sockets packets. First, the server starts a TCP listener with a given port and waits until a client connects. The client is started by the therapist by entering the IP address of the server. It tries to connect to the server with TCP. If the client receives a welcome message from the server within a short time period, it confirms it with another message, or otherwise the connection failed, and the therapist has to reconnect. But if not failed, and the server receives the confirmation, the connection is established and ready to use for sending data. Each side of the network consists, additionally to a main class, which handles the network connection, of a NetworkSender, a NetworkReceiver, a Server/ClientSend and a Server/ClientHandle class.

The NetworkSender gets the raw input data from the scene, which has to be sent, and prepares it for the sending process. The Server/ClientSend class takes the prepared data and converts it to a byte package using a bit converter, before it is sent over TCP or UDP. The Server/ClientHandle class of the other side then reads the package and converts it back to its original data format. Finally, the NetworkReceiver takes the unpacked data and calls the according components of the application to use this data as input.

Table 4.1.: Overview of the different data messages.

\begin{tabular}{|l|l|}
\hline Protocol & Data message \\
\hline TCP & Welcome message (Server-Client) \\
TCP & Welcome received message (Client-Server) \\
TCP & Avatar data message (Server-Client) \\
TCP & Audio messages (Both directions) \\
TCP & Instruction messages (Client-Server) \\
\hline UDP & Position \& rotation messages (Server-Client) \\
\hline
\end{tabular}


The network is used to send a handful of different data. The messages are listed in Table 4.1. It is either an avatar data message, the position and rotation of the headset, a control instruction from the therapist or an audio message. The avatar data is once sent at the start of the therapy from the VR-side and contains information of the avatar's body properties, the outfit and the hairstyle, which is then loaded on the therapist-side. To allow the therapist to see the movement of the patient, the tracked position and rotation of the VR headset are sent and updated during the complete therapy session. Since it is updated all the time, UDP is used to reduce the network time, and it is not crucial if one packet gets lost. Further, if the therapist changes the visibility or the mood of the avatar, activates the lip synchronization, or ends the therapy, a simple message is sent to the VR-side containing an instruction in the form of a string. The server compares the received string with a set of possible instructions and executes the according action. The last kind of data are the audio messages. Both applications have a voice chat implemented, which allows transferring voices over the network. Unity provides a microphone class, which records the input from a real microphone. The input is split into small junks of samples and converted to bytes. The set of samples is then sent over the network. The other side receives the audio messages and enqueues them into an audio queue. If there are enough samples ready, they are converted back and used to fill a new audio clip. Finally, the audio clip is added to the audio source of the application and is played.

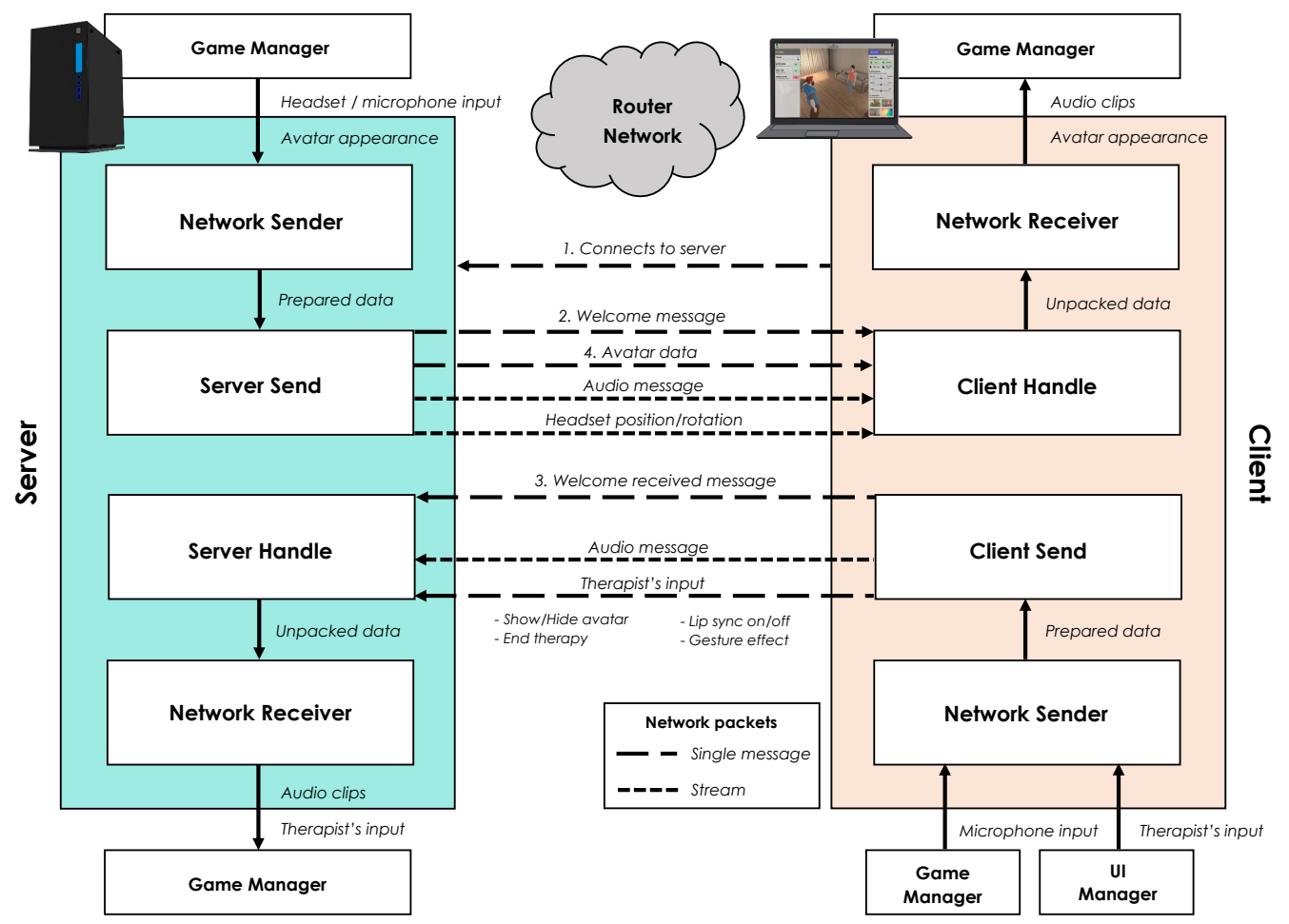

Figure 4.2.: Block diagram of the network protocol. 


\section{Implementation}

\subsection{VR-side}

The application on the VR-side consists of three different scenes: The start scene, the avatar customization scene and the main therapy scene. The main purpose of the VR-side is to provide the VR environment for the VR headset and the avatar customizer for creating an individual avatar. The final application is built as a Universal Windows Project, which is needed to run it on a WMR headset, like the HP Reverb. Additionally, a virtual reality SDK for WMR devices must be installed from the project settings.

After starting the application, the start scene is loaded on the screen. The VR-modus is disabled until the user starts a therapy. Figure 4.3 shows the start scene. It contains the start menu and presents a list of all available therapies (A). Each therapy represents a save slot in form of a small panel and shows the therapy number (an ID, to match a patient with its avatar), the date of the last session and a session counter. The therapy is saved as a simple text file on the disk containing this information and the UMA data of the avatar. A therapy can either be started, edited or deleted. To help find the correct therapy, two different sorting orders (B) are possible: Sorting by date with the current one first or sorting by the therapy number in ascending order. Additionally, a therapy number can be entered in a search bar (C), whereby the therapies are filtered in real-time. If there exists no therapy or the user will create a new one, a button for creating a new therapy (D) leads to the next scene, the avatar customization.

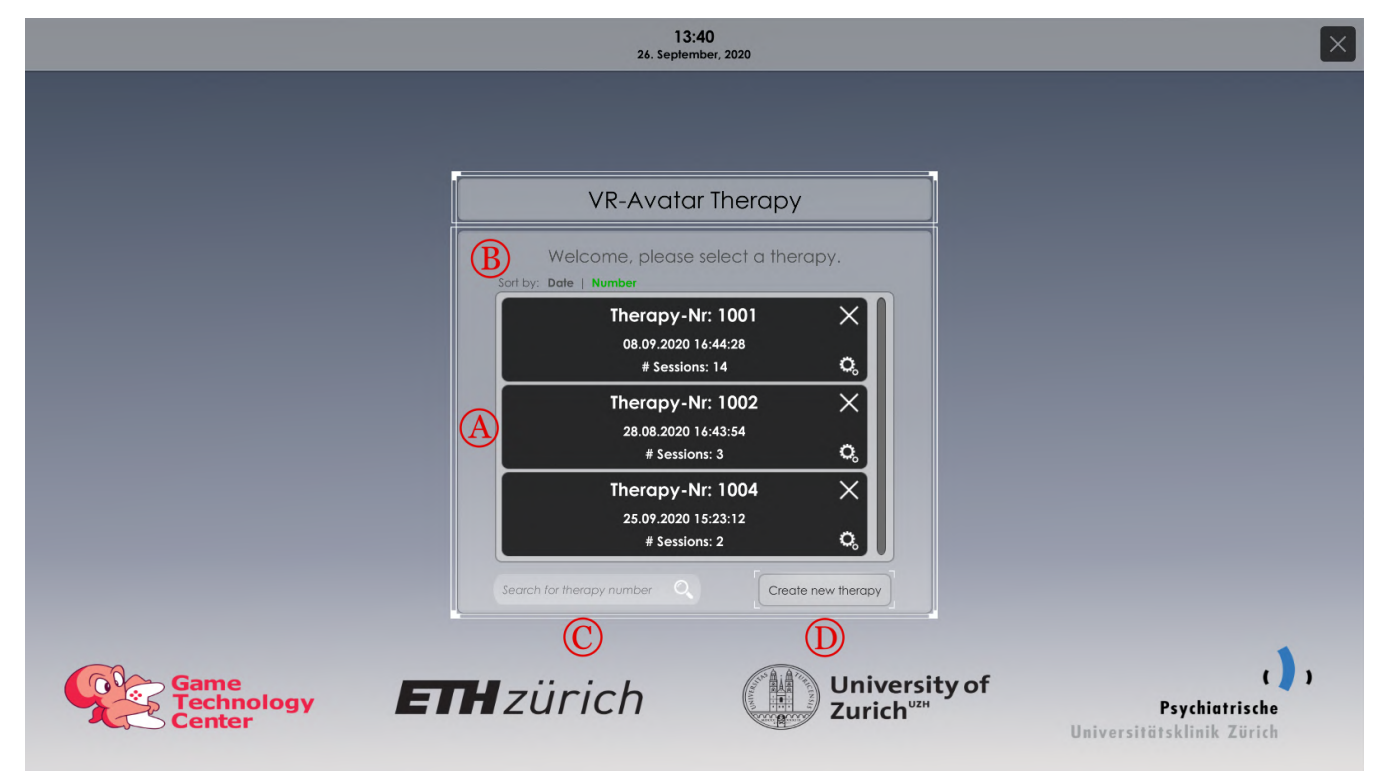

Figure 4.3.: Start scene showing available therapies. 


\subsubsection{Avatar customization}

After creating a new therapy or editing an existing one, a new scene of the application is displayed. It contains the avatar customization tool, to individually manipulate a 3D-character. The character itself is based on UMA [16], an open source package from the Unity Asset Store, which provides a system to create customizable characters. The packet comes among other things, with the base meshes for a male and female character, textures, a set of data for manipulating the avatar, an expression player and a wardrobe system with style recipes for adding cloth and hair. The data is divided into UMA DNA and a slot system. The UMA DNA allows manipulating more than 50 different body properties like arm length or feet size, which are represented by a value between 0 and 1 and can be set by code. The slot system of UMA allocates different wardrobe slots to the different body parts. Each slot can hold appropriate recipes, which store the data for an additional asset, like a shirt, a beard or pants. For this project, 8 different slots are used. The hair slot, the beard slot, the eyes slot, the chest slot, the leg slot, the feet slot, the shoulders slot, and the face slot.

The recipes are representing additional assets, which can be added to the avatar to change for example its outfit, hairstyle or face. The recipes store the appropriate slot, to which it will be added, the mesh and texture of the asset, and a shared color. The UMA packet comes with some predefined recipes for hair and cloth, but to increase the offer of different styles during the creation process, more assets are purchased from the Unity Asset Store. The UMA Hair Pack [19] contains 21 different hairstyles and 5 beards, the UMA Clothing Pack [17] includes 64 outfits from shoes to jackets, and the UMA Face Pack [18] comes with 20 different faces. Each of the packet consists of meshes, textures and the predefined recipes. To use the new assets, the recipes have to be added to the existing wardrobe system of the UMA character. All recipes are collected in an asset folder and can be accessed from the code with their name. To help the patient to select a desired hairstyle, beard or an outfit, for each recipe a small thumbnail is created, which shows the preview of the asset. Figure 4.4 and Figure 4.5 show a set of some available assets. 


\section{Implementation}

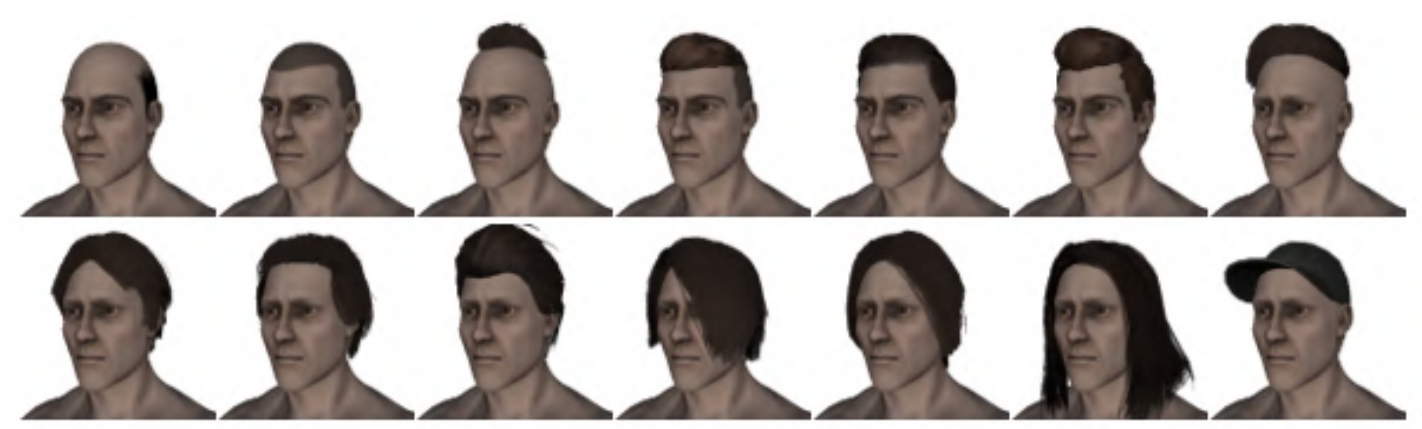

(a) Collection of male hairs.
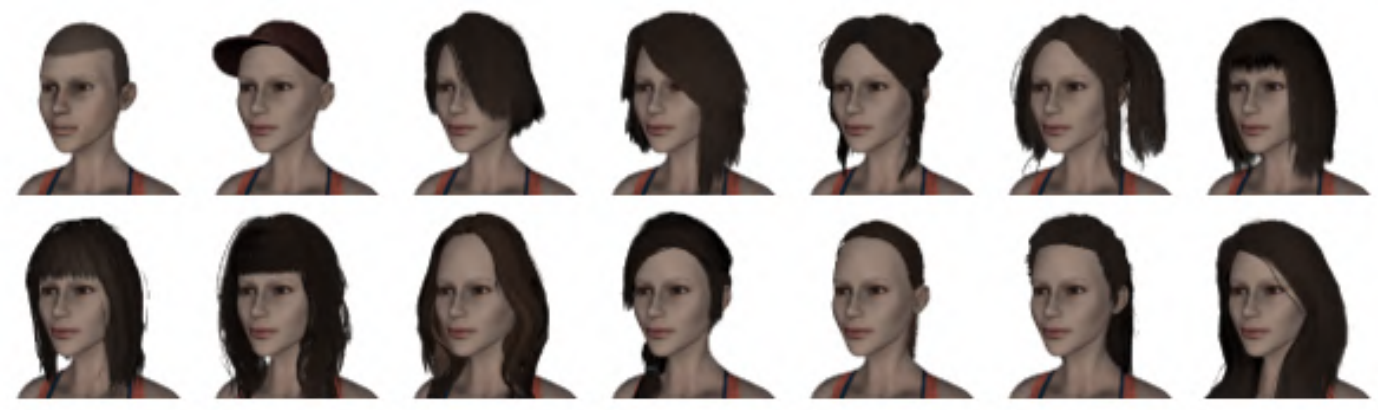

(b) Collection of female hairs.

Figure 4.4.: Collection of different hairstyles for UMA.

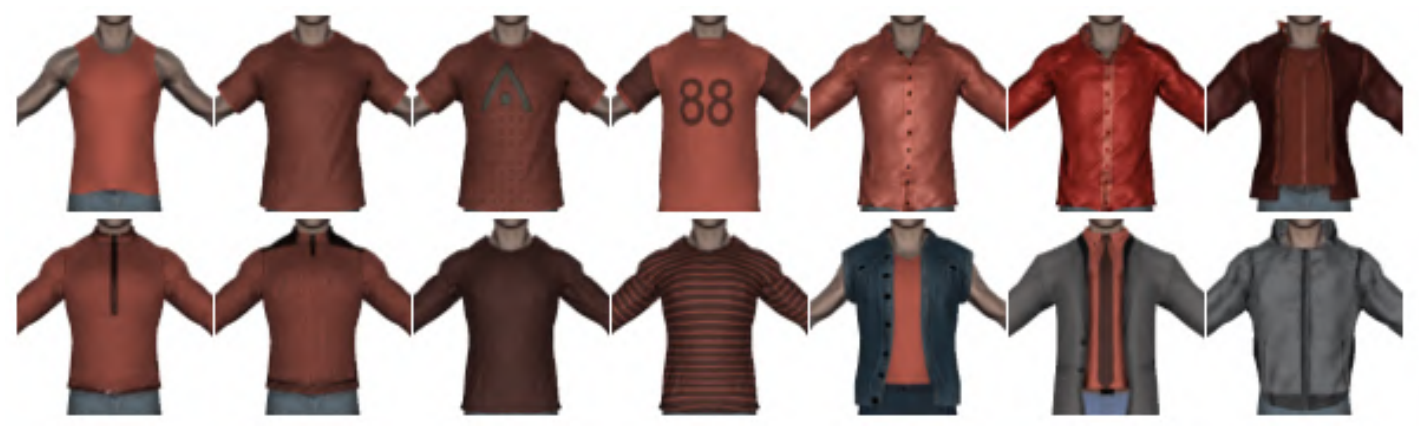

(a) Collection of male outfits.

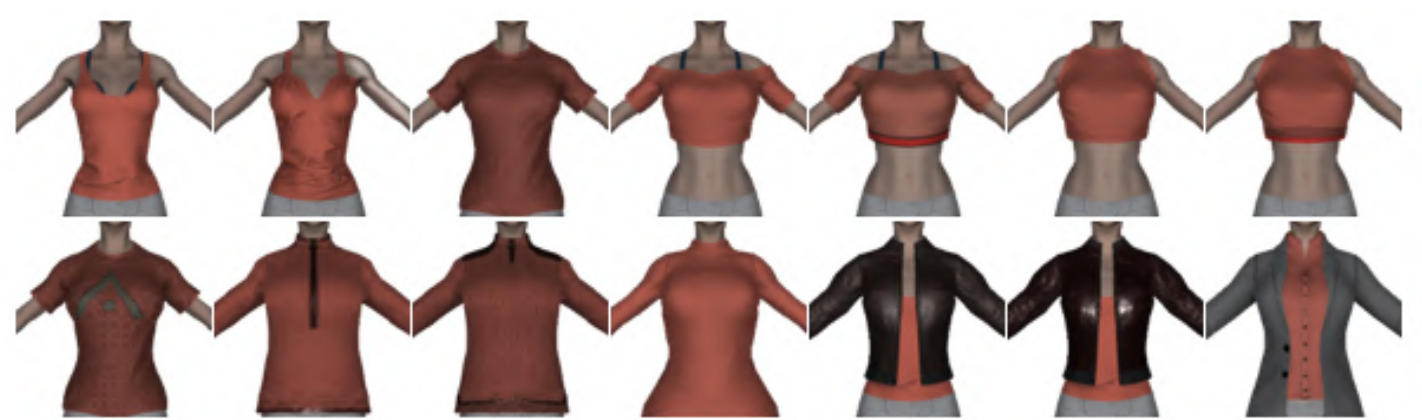

(b) Collection of female outfits.

Figure 4.5.: Collection of different outfits for UMA. 
In the first therapy sessions the patients work together with the therapist and create an own virtual avatar, representing as good as possible the entity to which they attribute their verbal hallucination. The creation phase is split into four different steps. This helps to increase the usability and separates the functionalities into different views. Each view provides in its center the avatar, which allows to see the changes in real-time. The features, which can be changed, are presented in a panel on the left and right side. These features are either a body or face property in form of a slider, a selection panel, where the patient can select a style for the outfit or hair, or a color button to change the appropriate color. Following, the four different steps are explained.

The first step allows the user to change the gender, either male or female, the skin color and 5 body properties for the male and 6 body properties for the female character. The body properties can be changed with a set of sliders, whereby each slider is connected to one or multiple UMA DNA properties and adjusts its value accordingly. The properties are the height of the avatar, the overall muscle structure, the body fat, the length of the arms, and the length of the legs. For the female avatar, additionally a slider to change the breast size exists.

The second step allows to change the face properties of the avatar. Since the focus of the avatar customization lies on the face, more sliders are provided than for the body. To better see the changes of the face, the camera zooms to the face. The possible changes are the size, the rotation, the horizontal spacing and the color of the eyes, the size, the vertical position and the curve of the nose, the size and rotation of the ears, the size of the lip and mouth, and the size of the jaw, chin and cheek. Additionally, the texture of the face itself can be changed. There are 8 possible faces, which allow to change the overall characteristic of the face.

After the second step is done, all body and face properties of the UMA are set. In step three and step four the style of the avatar can be changed. Step three provides a set of different styles for the hair, eyebrows and beard. Each category is presented as a selection panel, where all the possible styles of that category are shown in form of a small preview image. The user can select a style with the mouse, and it gets highlighted green. Internally, the name of the clicked object is used to find the according recipe in a recipe list. If the name matches, the recipe is loaded to the correct UMA slot and the avatar gets updated, showing the new outfit. There are in total 16 different hairstyles, 8 different eyebrows, and 8 different beards for both the male and female avatar. In addition, one single color button exists, to directly change the color of them at once.

In the fourth and last step of the avatar customization, the outfit can be chosen. There are again different selection panels for the different categories, but each category has its own color button. There are 12 outfits for the upper body, 8 outfits for the lower body, and 8 different shoes. Additionally, there are 3 options to add a jacket or a vest on top of the other outfit. But some outfits cannot be combined with a jacket and are blocked while wearing the jacket. Finally, if the patient is happy with the avatar, it can be accepted. The user is asked to enter a therapy number, and the avatar data is saved on the disk. The start scene is loaded again, and the new therapy can be found in the list. Figure 4.6 shows the process of creating an avatar in four steps. 


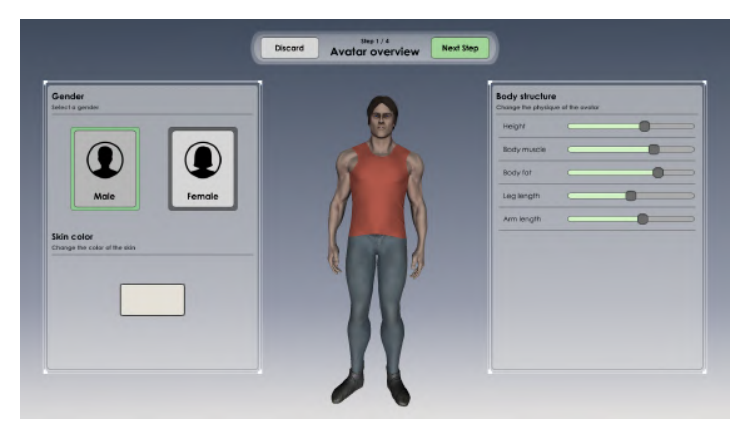

(a) Step 1: Body

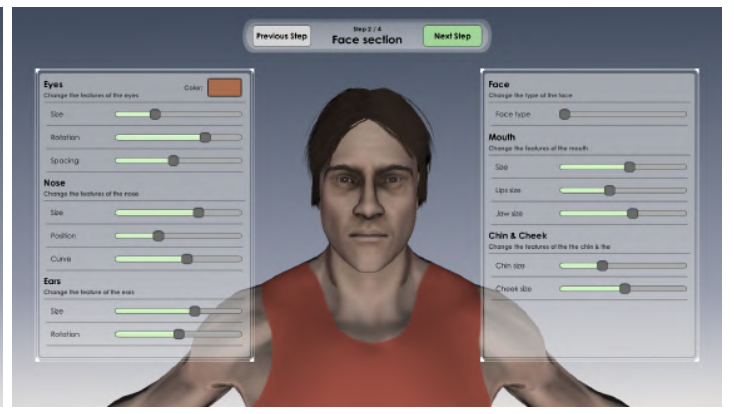

(b) Step 2: Face

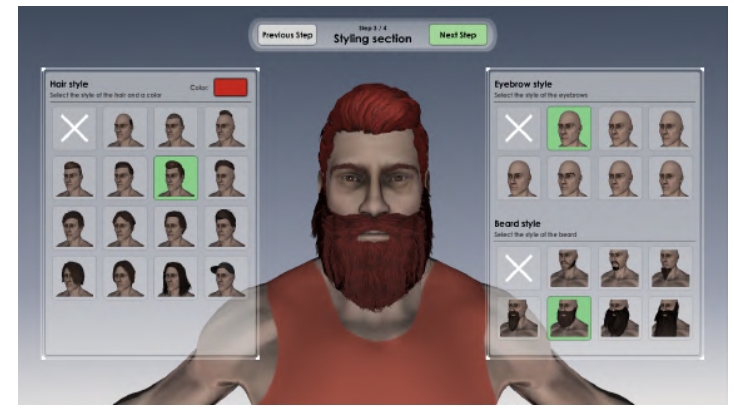

(c) Step 3: Hair

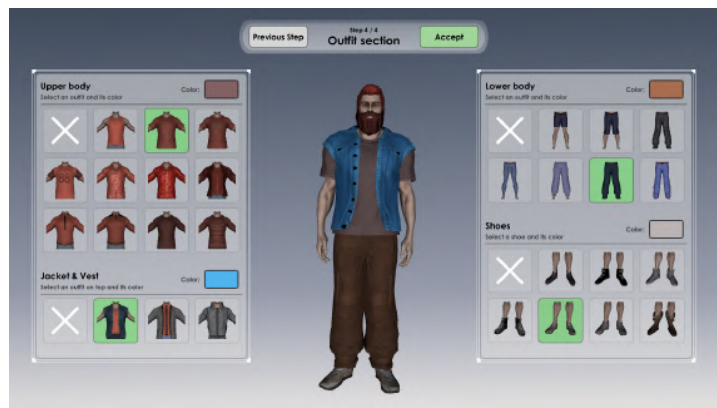

(d) Step 4: Outfit

Figure 4.6.: The four steps of the avatar customization.

\subsubsection{VR scene}

As soon as the user starts a therapy from the start menu, the main therapy scene is loaded, whereby the VR headset is enabled and starts showing the scene on its display. The scene is directly created in the 3D editor of Unity and needs at least a camera object tagged as main camera, to represent the view of the headset. For this project, the camera is set to show the therapy scene from a first-person perspective of the patient.

The idea of the scene is to look like a small and simple therapy room, where the patient stands in the middle of the room surrounded by a set of furniture. The scene consists of a room, a camera, some light sources, and a placeholder for the avatar. The room is designed as a rectangle box, where three sides are covered by walls and one with a big window, hidden behind a curtain. To keep the focus of the avatar, the curtain prevents the patient to look outside, but at the same time, some sunlight can shine through, which is simulated by a light source outside the box. The box is closed with a white ceiling and a wooden floor. To decorate the room, some pieces of furniture are placed, consisting of a sofa, a chair, a glass table, a wooden table, and three floor lamps. The models, textures and materials for the room and the furniture are purchased from the Unity Asset Store [1]. To adjust the form of the room, some meshes are edited with Blender (Version 2.79), an open source 3D creation tool. 
To create a realistic looking lighting in the room, different types and sources of light are used. A rectangle area light outside the window creates the impression of the sunlight and is baked into the scene, whereby Unity provides some quality settings to bake the light into light-maps. For this scene, the size of the light-map is 512 x 512 pixels. Additionally, a directional light and three point lights, attached to the floor lamps, illuminates the scene to make the room a bit brighter. The point lamps also create some soft shadows in real-time.

The complete scene is designed to increase the level of immersion for the patient. As soon as the patient wears the VR headset, the patient can look around $360^{\circ}$ and explore the room. If needed, the patient can also move around in the virtual room, as long as the real room provides enough space. After the first exploration phase, the avatar can be loaded, and the previously created avatar appears in front of the patient. The avatar is placed with some distance to the patient and near a wall, to not intimidate the patient with its presence. After that, the therapy is ready and the patient can start interacting with the avatar, which is remotely controlled by the therapist. Figure 4.7 shows the therapy room within the Unity Editor and shows the different lighting sources, the avatar placeholder and the furniture.

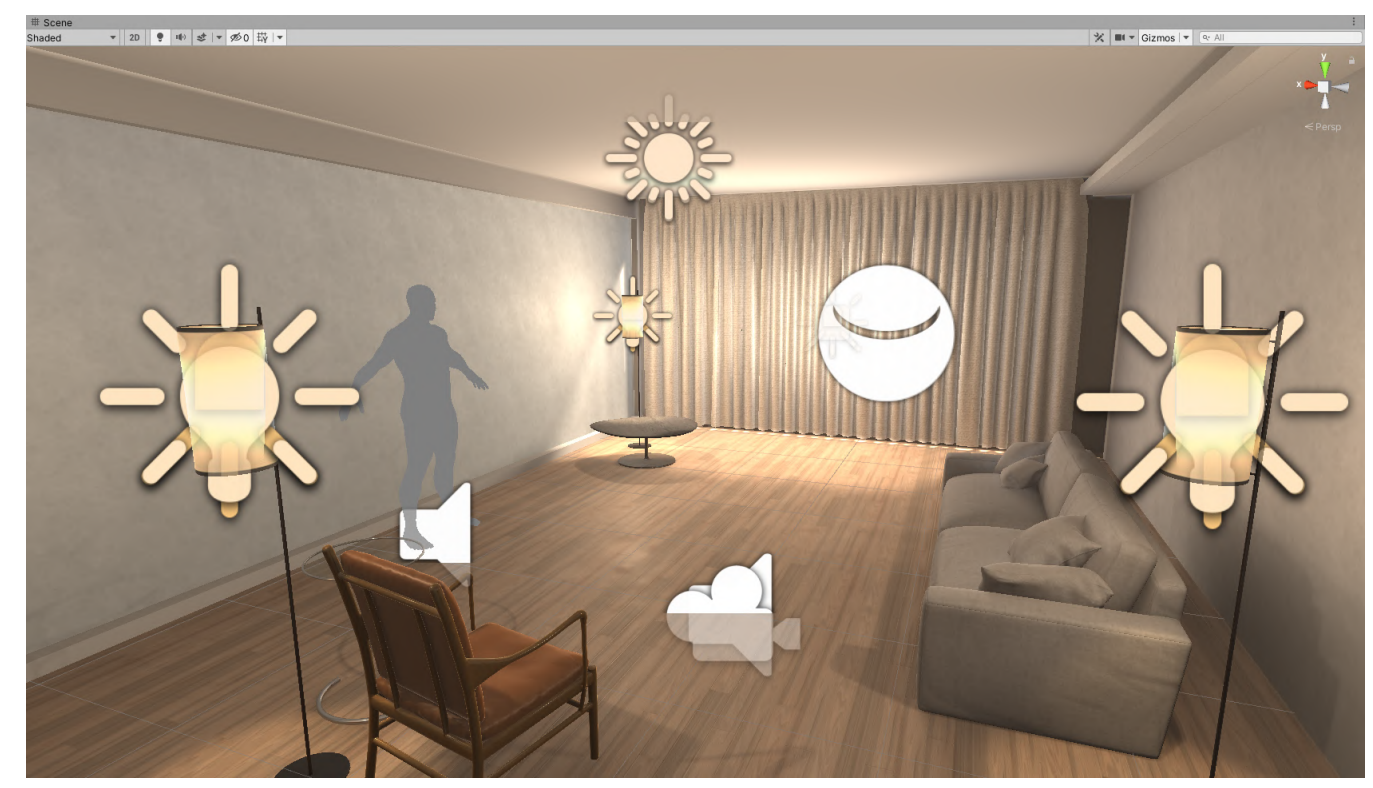

Figure 4.7.: View of the therapy room within Unity. 


\subsection{Therapist-side}

The second application is built to run on the laptop of the therapist. It allows the therapist to connect to the VR-side and remotely take control of the avatar. Furthermore, it provides the voice chat to talk to the patient and shows a copy of the virtual therapy room to have an overview of the therapy. The application consists of two scenes: A start scene and the therapy scene.

The design of the start scene is the same as for the VR-side. Figure 4.8 shows the start scene. This time, there is no list of the therapies, but an input field (A) to enter the IP address of the server. To speed up the process, the application remembers the last entered IP address. After pressing the connect button (B), a connection between the VR-side and the therapist-side is established like described in Section 4.1. In the top, the current time and date is displayed as well as an exit button, which closes the application.

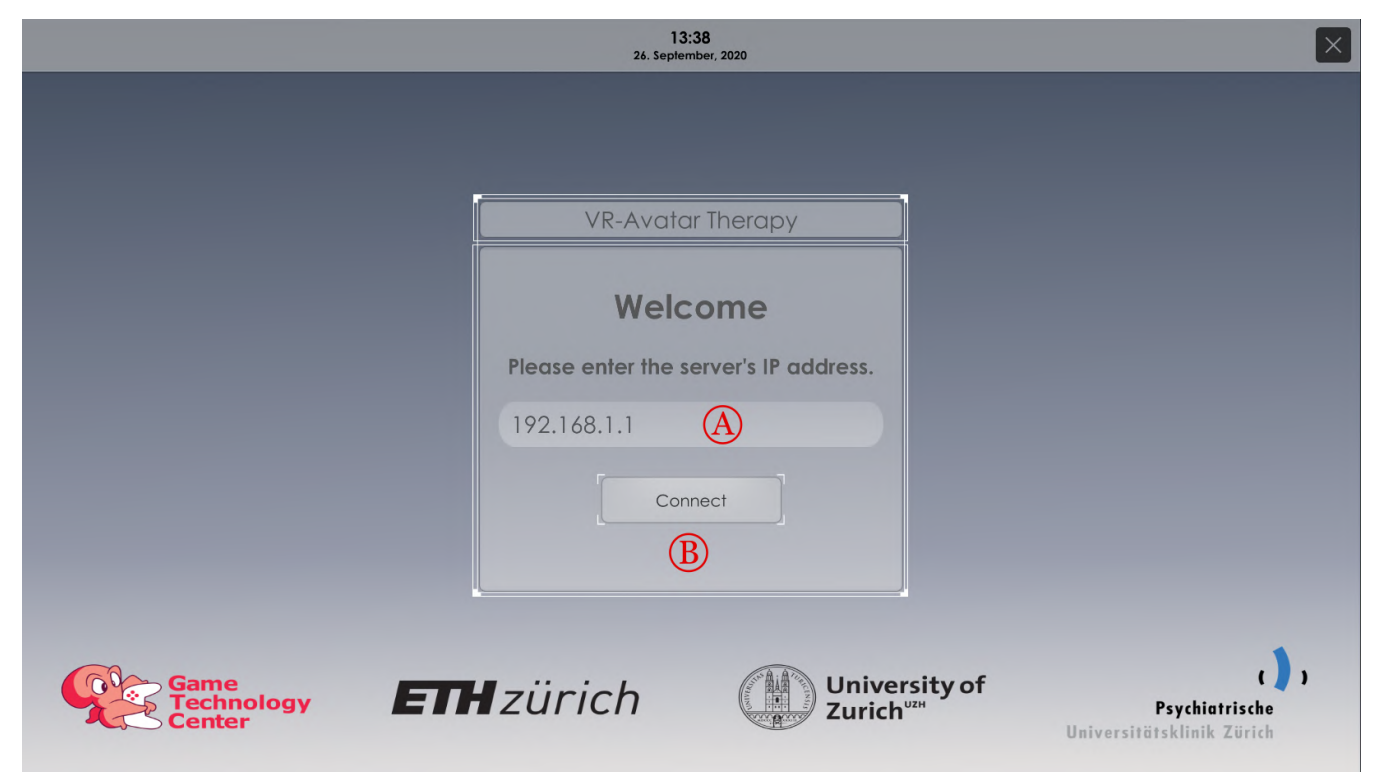

Figure 4.8.: Start scene asking for the IP-address.

If the connection is successful, the therapy scene is loaded. There is no need for a VR integration, since the therapist joins the therapy only with their laptop. First, the avatar data is received from the server and the avatar itself is prepared. The scene consists of the same therapy room as the VR-side, but an additional 3D character stands in the middle of the room. For this character, a default UMA is used, representing the patient. This character receives the tracking data of the VR headset from the patient and allows the therapist to see where the patient is looking at.

In addition to the option of monitoring the therapy, the therapist has access to a set of control options. Therefore, a GUI is provided, which shows a control panel on the right side. Figure 4.9 shows the blank view of the therapy with the open control panel. The panel consists of four different parts: A button for showing or hiding the avatar (A), a section to change the settings of the voice chat (B), a section to change the mood of the avatar (C), and a section to change the camera view (D). 


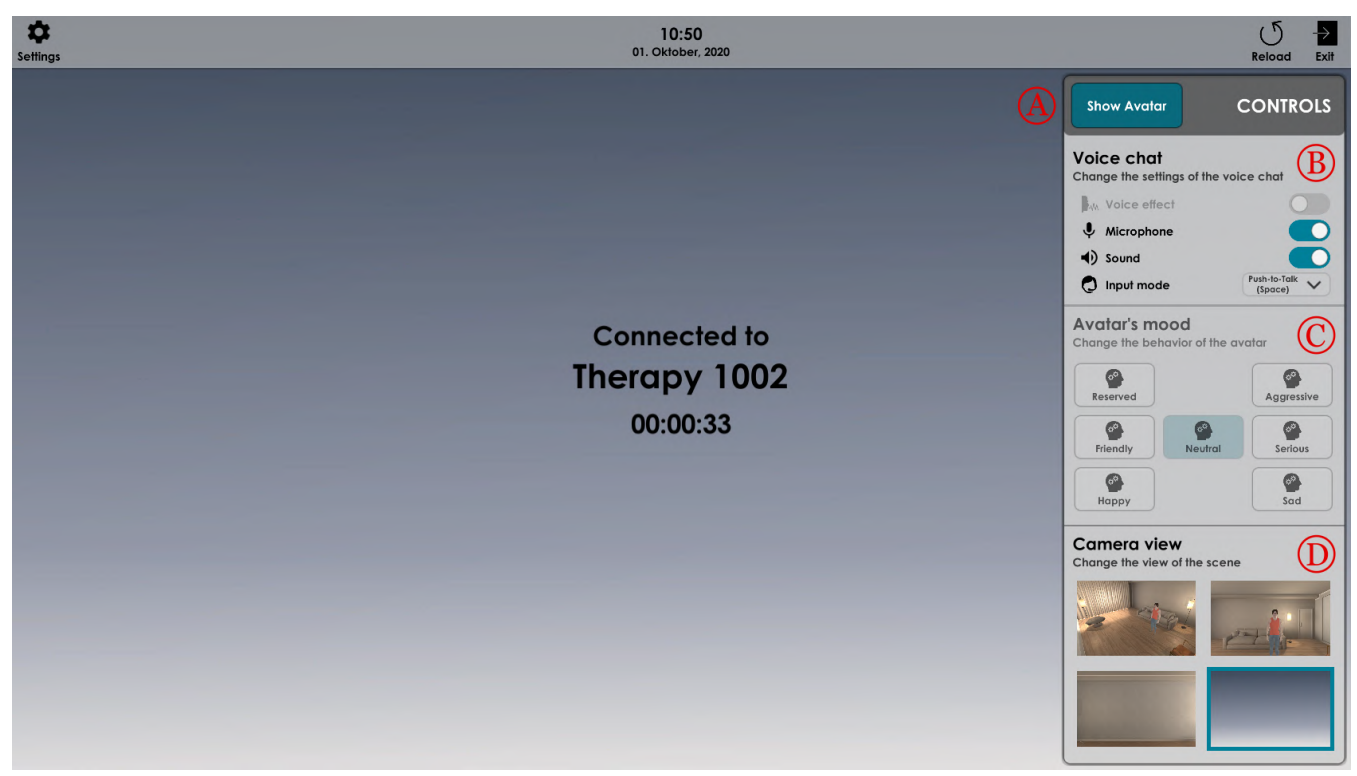

Figure 4.9.: Blank therapy scene showing the control panel.

The button for showing or hiding the avatar allows the therapist to enable or disable the avatar on both applications. When a therapy scene starts, the avatar is deactivated, which allows the patient to first explore the room itself. After that, the therapist can load the avatar with a click on the button and hide it again with another click. The voice chat settings provide a set of on/off toggles and a small drop-down list. The toggles are for muting the microphone, muting the sound input from the VR-side and activating the voice effect. The latter one is used to switch between the normal voice of the therapist and the transformed one, representing the voice of the avatar. The small drop-down list provides two different chat modes: A push-to-talk mode or a voice activation mode. If the push-to-talk mode is active, the voice of the therapist is only sent if the space key is simultaneously pressed on the keyboard. For the voice activation, no key has to be pressed as the voice is sent all the time. More details are explained in Section 4.3.1.

The section to change the mood of the avatar offers 7 different mood buttons. Each activates a different mood state on the avatar and only one can be active at the same time. The mood of the avatar is further discussed in Section 4.3.2. The last part of the control panel is the camera view. To give the therapist a better overview of the scene, three different cameras are placed in the scene. One shows a top view, to see the avatar and the character of the patient at once, one shows the view from the eyes of the avatar, and one from the eyes of the patient. The latter one allows the therapist to see the same as the patient currently sees through the headset. If the therapist wants to focus on the communication and do not need to see the room, the cameras can be disabled. A blank view is presented, which shows the therapy number and the total spent time in the therapy session. Each of this view is presented as a small preview image in the control panel and can be selected by clicking on it. Figure 4.10 shows the three different camera views and the blank one. 


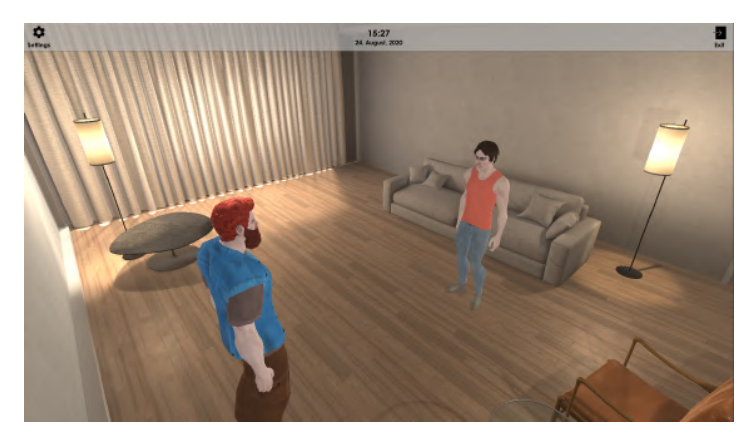

(a) Top view

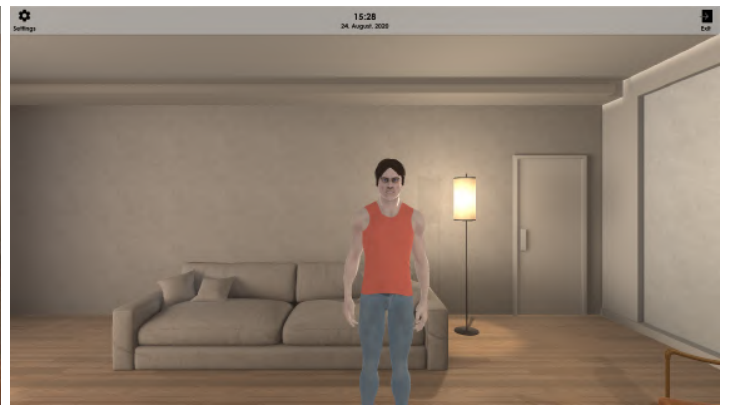

(b) View from the avatar

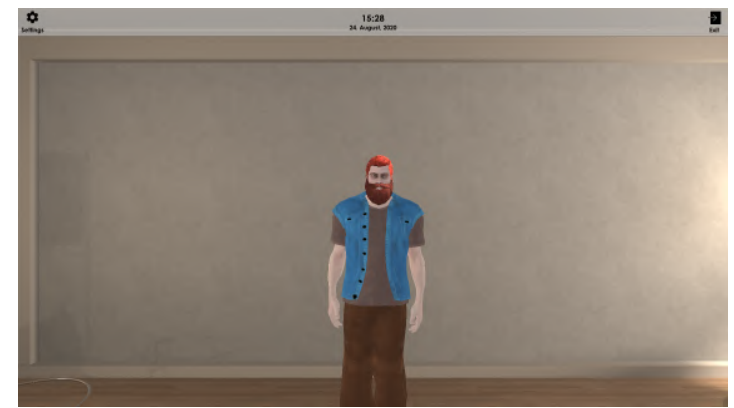

(c) View from the patient

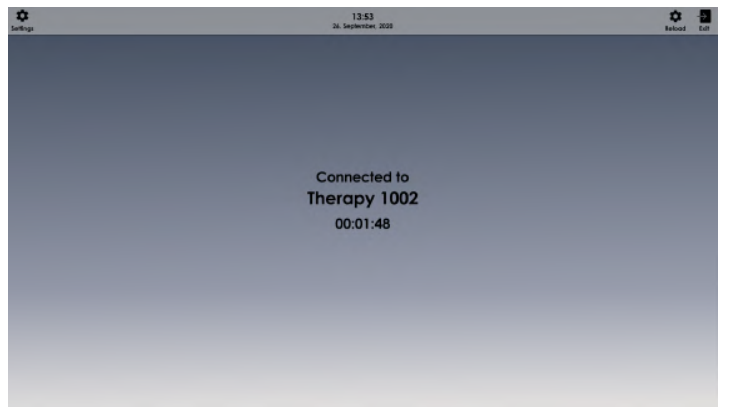

(d) Blank view

Figure 4.10.: Therapy scene from different camera views.

In addition to the control panel, the GUI provides a toolbar at the top, on which a setting button, a clock, a reset button and an exit button are placed. The setting button opens a second panel on the left side, where some additional options exist. The volume of the application can be changed, the control panel can be hidden, or a debug console can be showed. In the debug console some system outputs are printed, like information of the network connection or the attached microphone. The clock presents the current time and date and helps to control the length of the therapy session. Further, if something went wrong during the therapy, there exists a reset button, which starts the therapy again without stopping the VR scene for the patient. As a last option, the therapist can always close the therapy by clicking on the exit button. This ends the therapy session on both sides. 


\subsubsection{Voice modification}

The main form of the therapy is an interaction between the patient and the created avatar, where the patient speaks to the avatar, and the avatar can talk back, controlled by the therapist. The therapist has two options to speak to the patient, either with the normal voice or a modified version of it. The normal voice can be used to give the patient some instructions, ask for some feedback or explaining the situation. In this case, the patient hears the voice of the therapist directly and the avatar is not involved. But if the therapist wants to speak as the avatar, the voice gets transformed first. Therefore, the microphone input of the therapist goes through a voice modification device. For this project a Roland VT-4 voice transformer [11] is used. It provides a set of different options to manipulate a voice in real-time. Relevant for creating the avatar voice are the pitch and formant frequency, which can be set with sliders, and lead to a deeper or higher voice. In addition of creating a 3D character during the avatar customization, also the voice of the avatar is created by adjusting these sliders and saving the settings on the device. The Roland VT-4 provides up to 8 different save slots, which allows to easily save and load different voices. The device transfers the manipulated voice along with the clear voice directly to the laptop of the therapist via USB. Thus, the application gets two input signals, a mix (manipulated voice) and a dry (clear voice) signal. The voice effect toggle in the control panel switches between these two signals and sends either the manipulated voice or the clear voice.

To increase the impression that the avatar is speaking, also the lips and mouth of the avatar are moving. To achieve this, the lips are synchronized with the voice input. The SALSA package [12] from the Unity Asset Store allows such a synchronization. It provides a high quality, real time lip sync approximation for $2 \mathrm{D}$ and $3 \mathrm{D}$ characters and can be added directly to an existing UMA character. It gets access to the audio source, which is used to play the voice, and manipulates the lip and face properties of the avatar according to the intensity of the voice input. If the intensity of the input increases, more face bones are affected, and the lip movement increases.

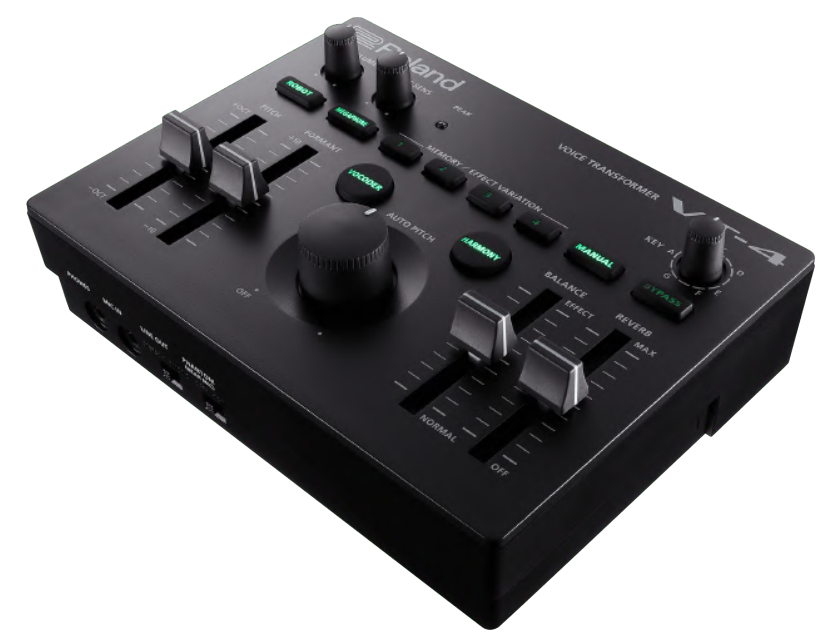

Figure 4.11.: Roland VT-4 Voice Transformer [5]. 


\section{Implementation}

\subsubsection{Gesture control}

In addition to the voice of the avatar, the therapist can also control the behaviour of the avatar. The control panel offers a set of different buttons, to change the mood of the avatar. With a simple click, the avatar changes the expression and movement behaviour of the avatar. There are 7 different mood states available: A neutral, a reserved, an aggressive, a friendly, a serious, a happy, and a sad state. Each provides an own set of gesture animations and a face expression for the avatar. An animation controller on the avatar handles the logic for playing the correct animation clips. It works with different animation layers, where the different clips can be placed and connected. For each mood state, 2 different layers exists. One controlling the animations for the upper body and one for the lower body. Only one pair of layers, responsive for the current mood, is active at the same time and plays a set of clips in a loop. The animation clips are downloaded from Mixamo [9], a platform for 3D character animations, and are chosen to represent the gestures for each mood. Additionally, each mood changes the face properties of the UMA DNA, to create a meaningful facial expression. Mostly the mouth and face properties are changed.

The overall idea to provide multiple moods, is to give the avatar more personal characteristics. The avatar, like a normal person, does not just stand still with a neutral face expression, but is able to move the body and parts of the face. This should increase the immersion for the patient as the avatar can show its feeling. A therapy session can maybe start with the avatar being aggressive, and over the course of the therapy turn into a more friendly state as the patient successfully interacts with the avatar to calm it. Figure 4.12 shows the different mood states of the avatar. 


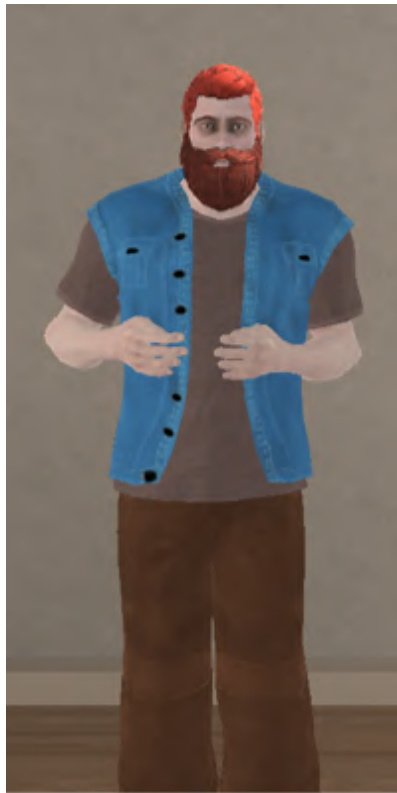

(a) Reserved

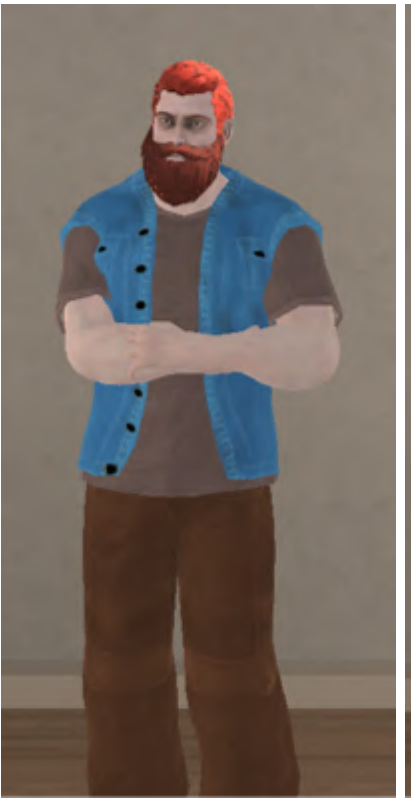

(d) Serious

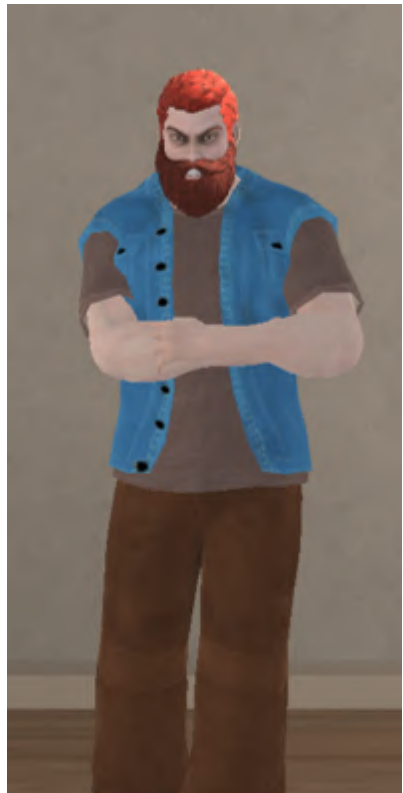

(b) Aggressive

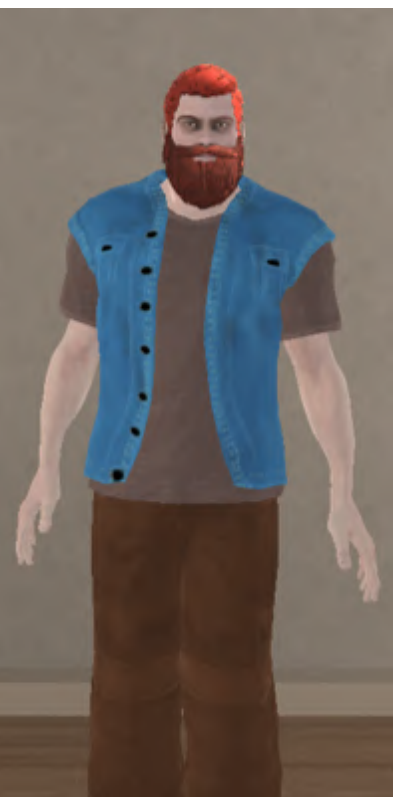

(e) Happy

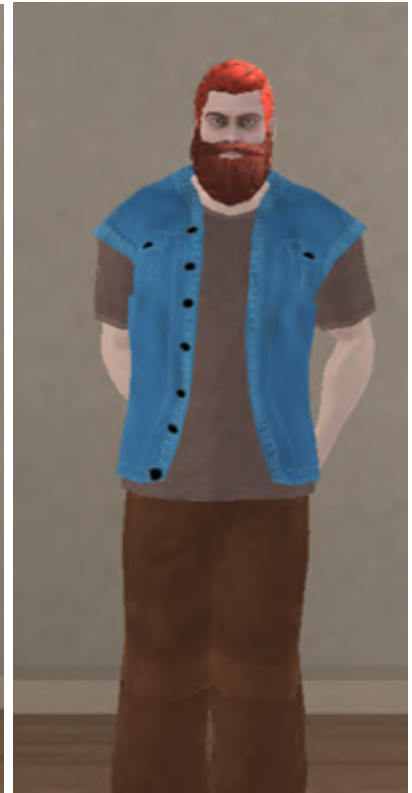

(c) Friendly

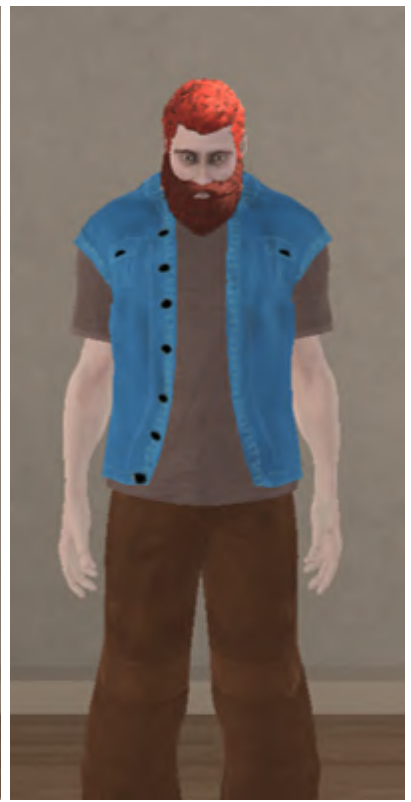

(f) $\mathrm{Sad}$

Figure 4.12.: Different mood states of the avatar. 



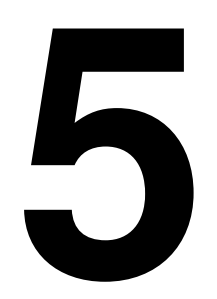

\section{Evaluation}

The finished VRAT system will be used by therapists at the PUK for a study, to treat people who actual hear voices. Therefore, it requires a high level of usability on both the VR application and the control application. It has to be easy to use and the patients, as well as the therapists, should have a good feeling by performing the new form of therapy. To achieve this, the system is tested regularly during the development process by possible end users, and their feedback is directly used to further improve it. At the end of the project, after presenting and explaining the finished system in a small workshop, the system is intensively used and tested from the clinical staff, and a usability test is made. The results from the testing phase and the final usability test are presented in the next sections.

\subsection{Testing phase}

The regular tests during the implementation of the system help to check the functionality and usability of the different parts. The system is installed at the clinic and some voluntary patients are recruited together with some therapists. In a first step, the avatar customization is tested by the patients, where they can freely create a representation of the entity to which they attribute their voice. As a second step, the headset and the VR environment is tested, where the patients have to wear the headset, explore the scenery and interact with the avatar. In a third step, also the control application of the therapist is tested by simulating a small therapy session between a therapist and the patient. The testers have enough time to play around and are asked to answer some predefined questions. This pilot study is a qualitative research of the system and the testers are free to give any answer to the questions. Figure 5.1 shows the timeline of the testing phase. It presents the number of participants per tested step and week. 


\begin{tabular}{|c|c|c|c|c|c|c|c|c|c|c|c|c|c|}
\hline Week & $\mathbf{1 - 1 0}$ & $\mathbf{1 1}$ & $\mathbf{1 2}$ & $\mathbf{1 3}$ & $\mathbf{1 4}$ & $\mathbf{1 5}$ & $\mathbf{1 6}$ & $\mathbf{1 7}$ & $\mathbf{1 8}$ & $\mathbf{1 9}$ & $\mathbf{2 0}$ & $\mathbf{2 1}$ & $\mathbf{2 2 - 2 6}$ \\
\hline $\begin{array}{c}\text { Avatar } \\
\text { customization }\end{array}$ & & 2 & 2 & 3 & & 2 & & 1 & & & & & \\
\hline $\begin{array}{c}\text { VR experience } \\
\text { Control center }\end{array}$ & & & & 1 & & 2 & & 3 & & 2 & & & \\
\hline $\begin{array}{c}\text { System's } \\
\text { usability }\end{array}$ & & & & & & & & & & & & & 76 \\
\hline
\end{tabular}

Figure 5.1.: Timeline of the testing phase.

For the avatar customization the patients have to change the avatar, to look like a possible representation of their voice. Each of the tested patient gets successfully through all four creation steps. The questions are:

Table 5.1.: Questions for testing the avatar customization.

\begin{tabular}{|c|l|}
\hline Number & Question \\
\hline 1 & Are there enough options to create your avatar? \\
\hline 2 & Do you think the creation was complicated? \\
\hline 3 & Do you like the overall appearance of the creator? \\
\hline 4 & Was it easy for you to find the correct settings? \\
\hline 5 & How was your feeling during the creation? \\
\hline
\end{tabular}

For the avatar creation process 10 tests are made with a total of 6 testers. In the first tests, the main comments show that the testers already have a good feeling during the creation process and like the overall appearance of the creator. But there are not enough options to create the avatar, and the UI is too complicated. Due to these feedbacks, the avatar customizer is further improved, and parts are reimplemented. Next tests show a more positive feedback for all 5 questions. The creation phase divided into four steps lead to an improved usability, and the creation is rated less complicated. The testers also like the additional content and are finally happy with the tool.

To test the VR scene, the VR headset itself, the virtual room, and the appearance of the avatar are tested. The testers are asked to wear the VR headset for ten minutes and to freely explore the scenery. None of the tested person has used VR before and it is their first experience at all. After the exploration, these questions are asked: 
Table 5.2.: Questions for testing the VR scene.

\begin{tabular}{|c|l|}
\hline Number & Question \\
\hline 1 & Was the headset comfortable for you? \\
\hline 2 & Do you like the optic of the therapy room? \\
\hline 3 & Was the avatar realistic enough for you? \\
\hline 4 & How was your overall feeling? \\
\hline
\end{tabular}

The same 6 testers are recruited and 8 tests are made. A summary of the answers shows a quite positive feedback. The avatar and the room look realistic and good. The VR experience makes fun and the testers feel comfortable. Additionally, the headset setup is easy and fast, but after ten minutes, the testers are also glad to remove the headset. Some testers feel intimidated during the first tests, because of the movement and position of the avatar. By repositioning the furniture and the avatar, and adding more natural gestures, this feeling is reduced.

To check the functionality on the therapist-side, like the control panel and the voice chat, different testers with more or less experience with other computer software, are asked to use the application. They test the functions by simulating a small therapy session and are asked afterwards to give a small feedback:

Table 5.3.: Questions for testing the therapist-side.

\begin{tabular}{|c|l|}
\hline Number & Question \\
\hline 1 & Do you like the overall appearance of the application? \\
\hline 2 & Was it complicated for you to use the control panel? \\
\hline 3 & Was it easy to use the voice chat? \\
\hline 4 & How was your overall feeling? \\
\hline 5 & Do you think you can perform a complete session alone? \\
\hline
\end{tabular}

For this application 12 tests are made with 8 different testers, which simulate the role of the therapist. The overall feedback is good and all the participants like the overall appearance of the application. Most of the settings are easy to use, but the voice chat settings, in more detail the activation of the voice modification lead to a lot of complications. It is not clear which voice is active, since the therapists do not hear themselves. In addition, the extra voice transformer has to be used, which makes it even more complicated. Therefore, the UI is further improved to make it more consistent and clearer. The following usability test shows the final feedback of both applications. 


\section{Evaluation}

\subsection{Usability}

To check the usability of the application, a System Usability Scale (SUS) [15] is used, a tool for testing the usability of a system, created by John Brooke in 1986. It is an easy-to-use and quick questionnaire with a proven concept for industry standards. The SUS provides a set of 10 different questions, which can be ranked by the user from 1 to 5 . A 1 means, that the user does not agree on this point and a 5 means the user fully agree. The 10 Questions are:

Table 5.4.: Questions of the System Usability Scale.

\begin{tabular}{|c|c|}
\hline Number & Question \\
\hline 1 & I think that I would like to use this system frequently. \\
\hline 2 & I found the system unnecessarily complex. \\
\hline 3 & I thought the system was easy to use. \\
\hline 4 & $\begin{array}{l}\text { I think that I would need the support of a technical person to be able to use } \\
\text { this system. }\end{array}$ \\
\hline 5 & I found the various functions in this system were well integrated. \\
\hline 6 & I thought there was too much inconsistency in this system. \\
\hline 7 & $\begin{array}{l}\text { I would imagine that most people would learn to use this system very } \\
\text { quickly. }\end{array}$ \\
\hline 8 & I found the system very cumbersome to use. \\
\hline 9 & I felt very confident using the system. \\
\hline 10 & I needed to learn a lot of things before I could get going with this system. \\
\hline
\end{tabular}

The questionnaire is built in a way that the odd numbered questions should be ranked as high as possible and the even ones as low as possible to reach a high score of usability. To get a comparable score out of this questionnaire, the results are converted in a special way. For each odd question, 1 is subtracted from the result and for each even question, the result is subtracted from 5. Thus, each question gives a maximum of 4 points. All the points are summed leading to a result between 0 and 40 . To make this more comparable, it is multiplied by 2.5 , leading to the end score between 0 and 100. Systems with an average SUS score over 68 are considered suitable for use.

At the end of the project, the system is tested by the clinical staff with several professional backgrounds. They are asked to simulate both sides of the therapy and to fill out the provided questionnaire. Overall, 76 different tests are made; 38 for the therapist application, and 38 for the VR application. The testers have to fill out their gender, age, profession, and their ranking of the ten questions. The age of the testers is between 17 and 56 years and $74 \%$ are female and $26 \%$ are male. The participants are either a medical doctor, a therapist, a psychologist, a nurse or work in the administration. 
The questionnaires are collected and analysed with Microsoft's Excel. Figure 5.2 shows the final score for each question. The results for the VR application and the therapist's application are separated.

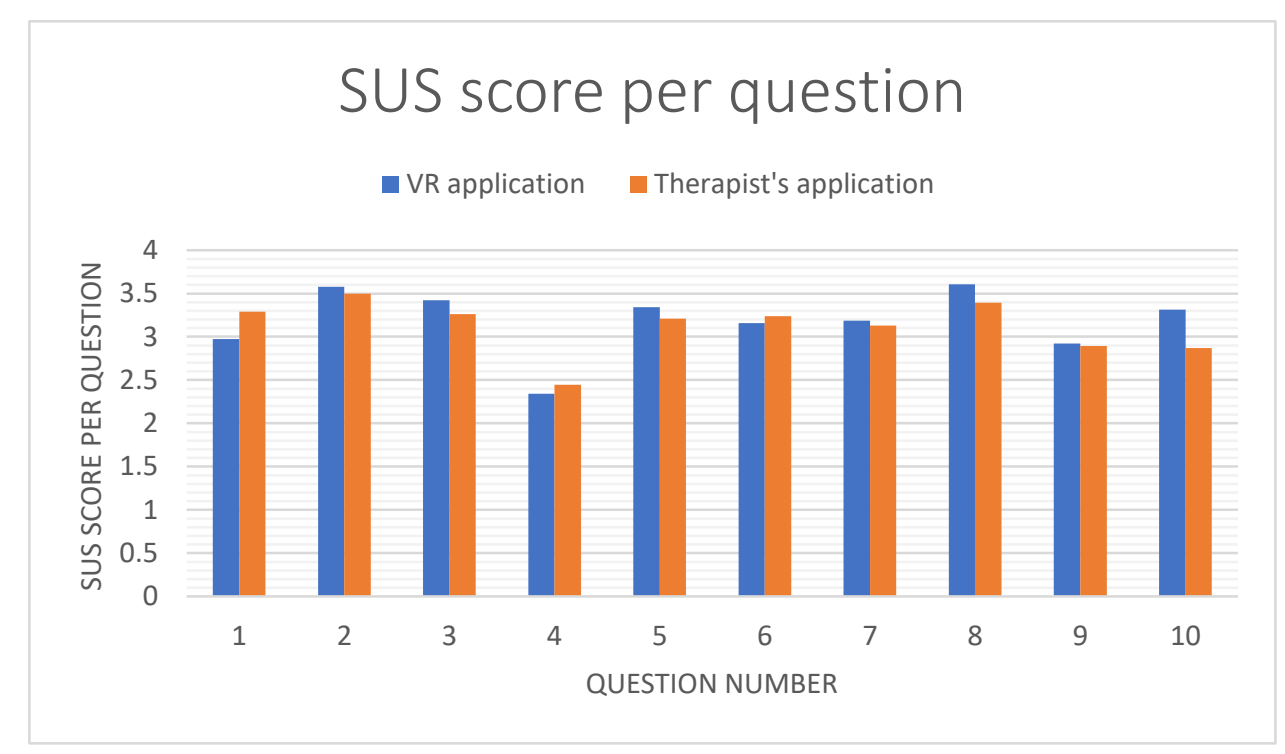

Figure 5.2.: SUS score per question.

There exists no big difference between the different applications, but the average ranking of the VR application is slightly higher (3.18) than the therapist's one (3.12). There are also no big differences between the scores of each question, except for question 4. It is noticeably lower as the others for both applications. The questions ask if a support of a technical person is needed. The complete VRAT is a highly novel approach and comes with an advanced technical setup. For most of the testers it is the first use of VR. Moreover, there are a lot of different components, which have to be combined. These factors can lead to the wish of some support, and it makes sense to give the therapist time before an actual therapy, to test the system and get familiar with the technical components.

To get the final SUS score, the points for each question are summed and multiplied by 2.5 . Again, the two applications are separated. Figure 5.3 shows a histogram of the individual sus scores. 


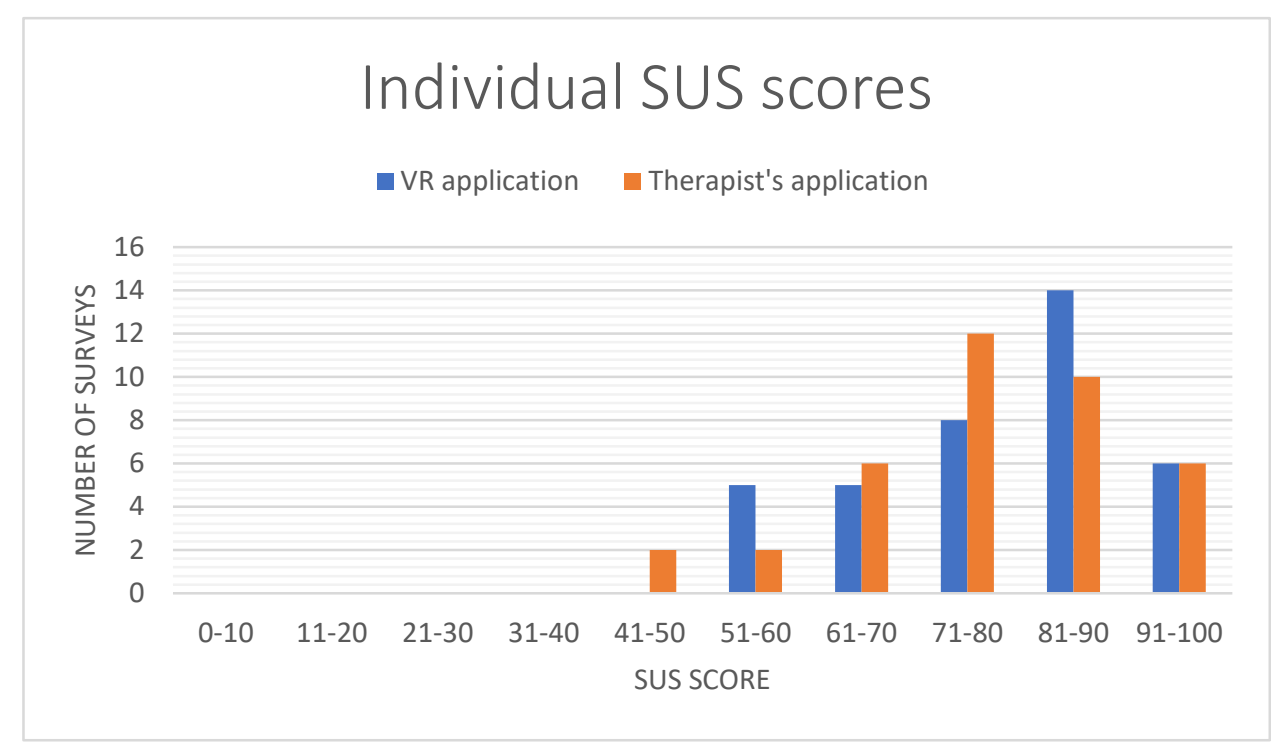

Figure 5.3.: A histogram of individual SUS scores.

In total, there are 38 results for the VR application and 38 results for the therapist's application. The histogram shows the distribution of the scores. The most values are around 80 for both applications, whereby the VR application is higher rated. To compare the final sUS score better, a detail view of the score itself is shown in Figure 5.4.

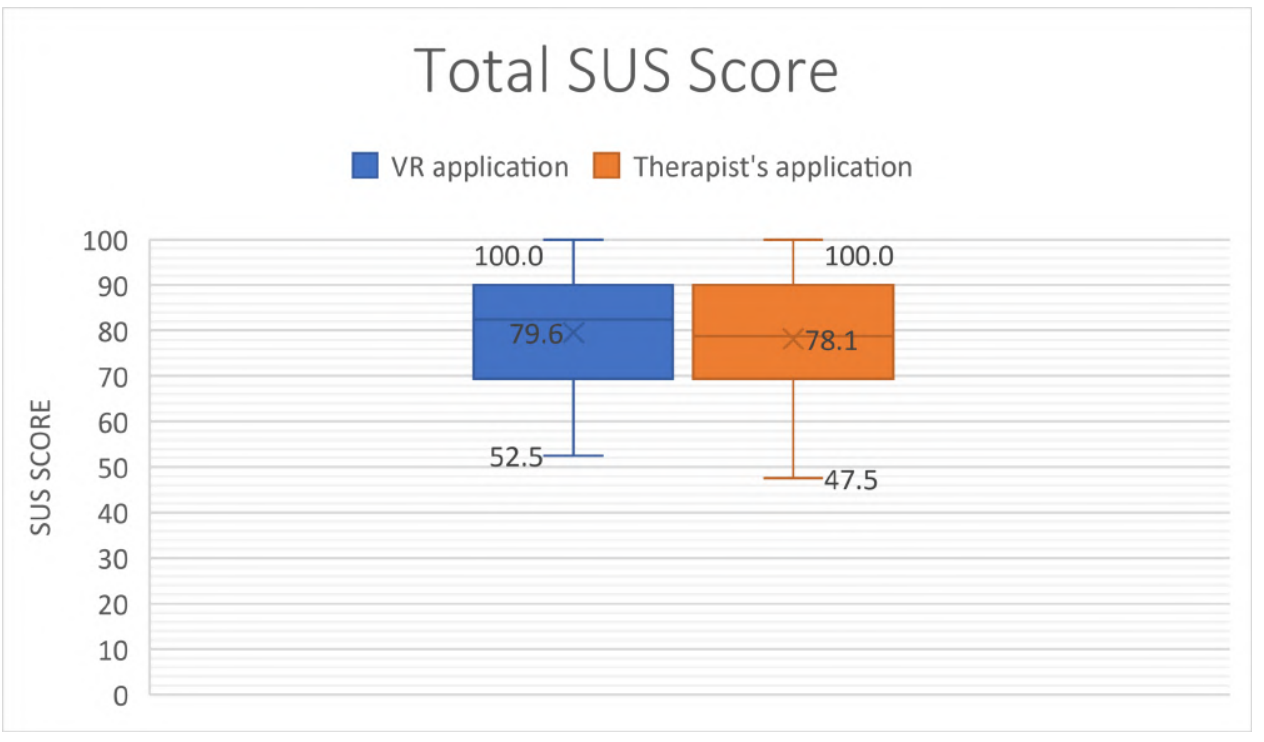

Figure 5.4.: Total SUS score for both applications.

Both applications are directly compared in a so-called box-and-whisker chart. It plots the minimum, the first quartile, the median, the third quartile and the maximum of the data set. In addition, the mean value is highlighted, which represents the final score. The scores for the VR 
application range from 52.5 to 100 with a median at 82.5 and a mean score of 79.6. For the therapist's application, the scores range from 47.5 to 100 with a median at 78.75 and a mean score of 78.1. In total, the complete system gets a SUS score of 78.85 out of 100 .

The final score can be used to interpret the overall usability of the system and indicates if a system is acceptable or not. In an article from 2008 about an empirical evaluation of the System Usability Scale [23] a comparison of mean SUS scores is presented and interpreted. Figure 5.5 shows this comparison, whereby the green bar represents the achieved score of this project. Both components rank between the Good and Excellent mark, yielding a highly acceptable system. The lower scoring of the Therapist's application could be influenced by the more advanced skills required to actually perform the therapy besides the technical control of the system.

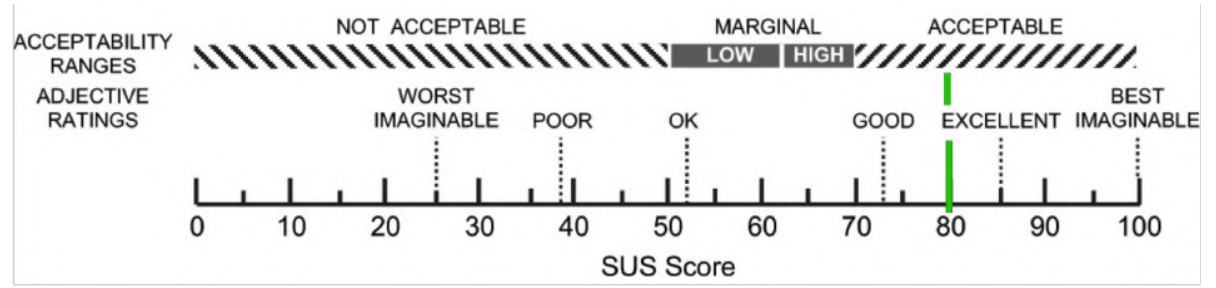

Figure 5.5.: Comparison of mean SUS scores [23].

In addition to the overall result, the SUS scores of the different profession groups are compared. The therapists, psychologists and medical doctors are combined in one group, since each group alone has not enough data to show an accurate result. Figure 5.6 shows the comparison of the professions for the VR application, and Figure 5.7 shows the comparison for the therapist's application.

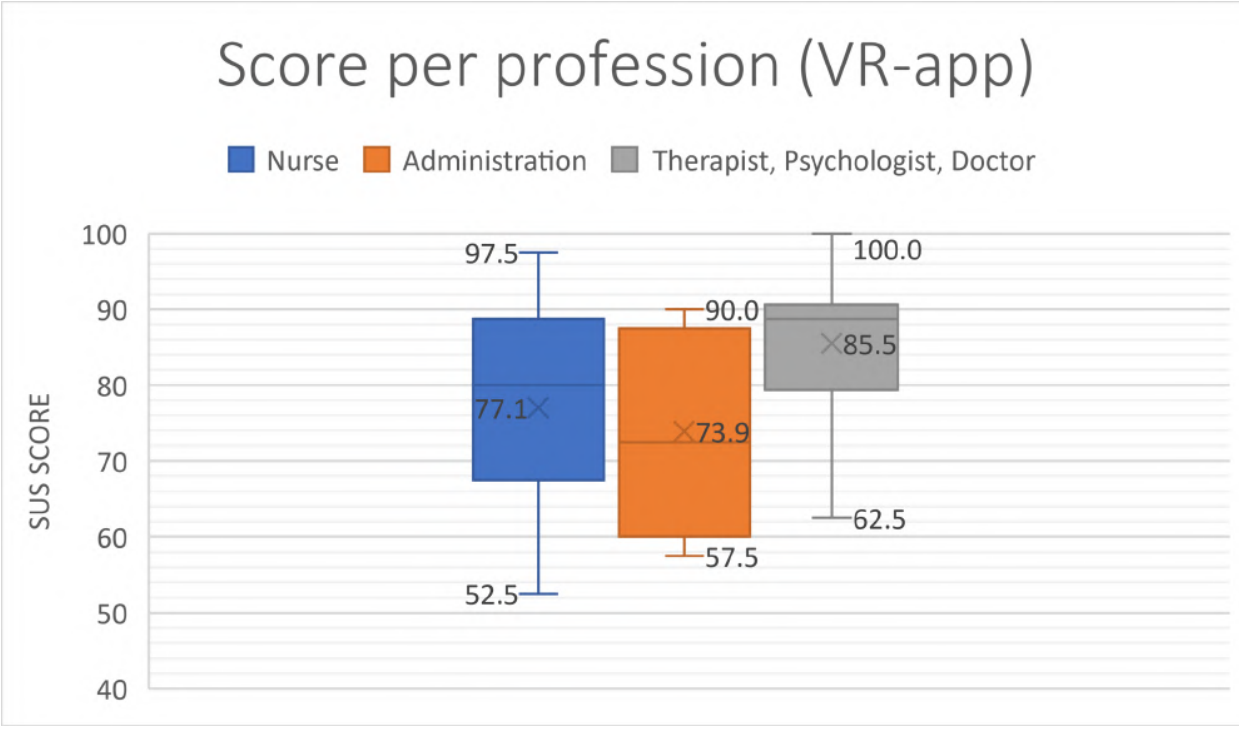

Figure 5.6.: SUS score per profession - VR application. 


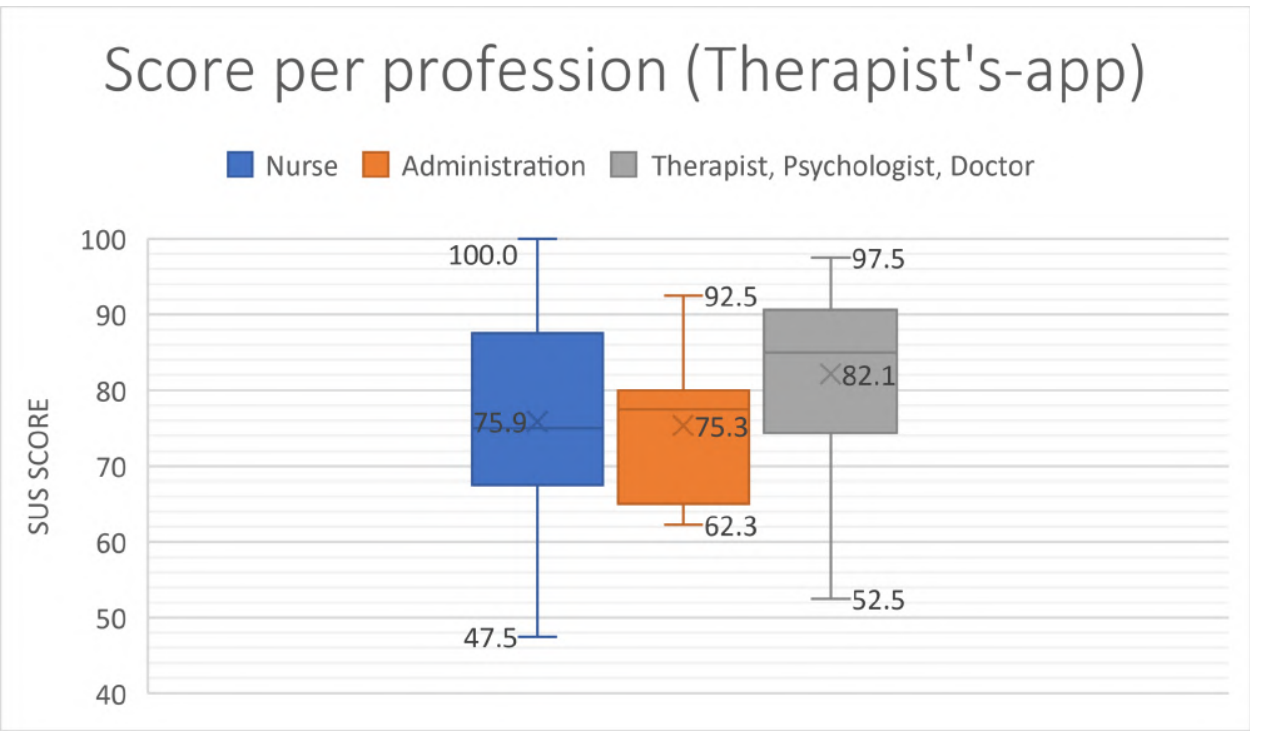

Figure 5.7.: SUS score per profession - Therapist's application.

For the VR application the SUS score of the nurses is 77.1 , the score of the administration staff is 73.9, and the score of the therapists, psychologists and doctors is 85.5. The therapist's application has the same trend. Here, the nurses rank the system with a SUS score of 75.9, the administration staff gives a score of 75.3, and the score for the combined group is 82.1.

The staff from the administration group ranks the system with the lowest score. Probably because they are not trained to lead a therapy, which they have to simulate during the test. But the actual end users of the system are the therapists, who are in the group with the highest SUS score. This is a good sign of having a ready and usable system for the target group. To make an even more detailed comparison of the individual professions, more tests of each group are needed. 


\section{6}

\section{Conclusion}

The previous studies from Montreal and London show promising results of using a new treatment form for people hearing voices. They provide an interaction between their participants and their voices by adding a visual representation of the voice to the therapy. Their findings show a high reduction in the intensity of hearing the voices and an improvement in the quality of life for affected people. In this project, the interaction with the visual representation of the voice, called avatar is even more increased by using a more advanced VR setup. The patient can immerse into a virtual therapy room and can interact with a full 3D avatar. Lip synchronization and different moods for the avatar increases the feeling of talking to a real person and enhancing the experience. Further, the detailed avatar customization process helps the patients to create a good approximation of a humanoid representation of their voice. The avatar itself is remotely controlled by a therapist. The therapist can speak through the avatar using a modified voice, provided by a voice transforming device. In addition, the therapist can also change the mood of the avatar, to represent the current condition of the avatar.

The usability test in form of a SUS shows an overall highly acceptable and user-friendly system. The final SUS score of 79.6 for the VR application and 78.1 for the therapist's application are an indicator for a good system [23]. The results of the used questionnaire also hint that therapists should become familiar with the technology and the therapy itself, before using the system and lead a complete VRAT.

The continuous feedback and the result of the SUS are very promising and show a high acceptance and feasibility of a VRAT in patients with acoustic hallucinations. The tested patients are highly motivated to use the new therapy, and the therapists are confident to use it. The implemented system provides all technical components for the new study at the PUK and is ready to use. 


\section{Conclusion}

\subsection{Lessons learnt}

During the development of the system, different design and implementation decisions are made. There are parts which work directly and others which can be improved. First, the modification of the voice is done with a voice transforming device. This adds an extra component for the user, which has to be installed and handled correctly. Also, it cannot be controlled via code, which makes it harder to use in the application. It would be interesting to test a voice modification software, which can be integrated into the application, rather than an additional hardware component.

Another realization is the importance of a consistent design concept for the UI. Using well known design principles helps to increase the usability and helps leading the user through the application. This contains the use of appropriate UI-elements, button icons and the arrangement on the screen. During the project, the UI is adjusted several times to increase the usability.

Further, the integration of existing animation clips from the web and combining them to a set of animations needs the luck of finding directly all the desired clips. If the animations are created internally, it would be easier to accurately fit the different mood states of the avatar.

There exist also components which are recommended to use, like Unity, which is used to build both applications. It works well to create a VR application and Unity makes it easy to integrate different assets into the same project. The editor helps a lot for developing, and among other things, the UI tool, the lighting tool and the animation tool facilitate the work. There exists a good documentation and enough examples. Further, also the HP Reverb headset does a good job. The quality is excellent, the setup is easy, and the integrated microphone and headphones work well.

Finally, the importance of the continuous testing with real patients and therapists, and the final usability test, should also be mentioned. The feedback helps a lot to improve the system, makes it more usable for the real end users and leads to discussions about additional features. The testing should start a soon as possible and in a regular time interval, to get feedback throughout the complete process.

\subsection{Future Work}

This project is a novel solution and a good starting point for testing the effect of using a VRAT for people hearing voices. The system provides a lot of functionalities and allows to perform a complete therapy session. But additional features and testing other variants should be considered in future works. Over the course of the thesis, different ideas are collected for creating new functionalities or rethinking existing ones. The following points could be interesting to further investigate.

1. A variant for the Avatar Therapy which is interesting to consider is the use of AR, where the patients can interact with a virtual representation of the entity behind their voice in a real room. This could help to give the patient the illusion to talk to a real avatar and would make the creation of a virtual room unnecessary. 
2. Not only the immersion should be considered. Also, the usability of the system is an important factor. The adaption for a VR smartphone-app, which can be used together with a cardboard system, increases the usability and lowers the setup time and the acquisition cost. The immersion is decreased, but the therapy can be used at multiple places or even at home for a remote therapy.

3. Another interesting topic is the creation of a new AI therapy, where the computer plays the role of the therapist. This allows a therapy session from home at any time and helps also patients, who cannot participate in a clinic. But for a new AI therapy, also the acceptance and efficiency of AI in therapies should be tested.

4. A direct improvement of the existing system is a more detailed lip synchronization. In this project, the lips are synchronized with the intensity of the voice input. A synchronization which also interprets different letters, words and phrases can give a better impression of a real talking human.

5. To completely use the technology of VR, a version where the patient and the therapist both interact in a virtual world could be tested. The therapist could use a headset and motion controllers to puppet the avatar in an advanced way. Therefore, a larger setup would be needed, which would increase the cost and setup time, but the immersion for the patient and the therapist would be enhanced. 



\section{$\mathbf{A}$}

\section{Appendix}

Table A.1.: Comparison of current VR headsets - Tethered.

\begin{tabular}{|c|c|c|c|c|c|c|c|c|c|}
\hline Name & Producer & Display & FOV & Resolution & Rate & Tracking & Platform & Date & Price \\
\hline Acer AH101 & Acer & LCD & $95^{\circ}$ & $2880 \times 1440$ & $90 \mathrm{~Hz}$ & Inside-out & WMR & 2017 & 299 \$ \\
\hline Fove & Vove Inc. & OLED & $100^{\circ}$ & $2560 \times 1440$ & $70 \mathrm{~Hz}$ & Eyetracking & SteamVR & 2017 & $599 \$$ \\
\hline HP Reverb & HP Inc. & LCD & $114^{\circ}$ & $4320 \times 2160$ & $90 \mathrm{~Hz}$ & Inside-out & WMR & 2019 & $599 \mathrm{CHF}$ \\
\hline HTC Vive & HTC & AMOLED & $110^{\circ}$ & $2160 \times 1200$ & $90 \mathrm{~Hz}$ & Outside-in & SteamVR & 2016 & $800 \mathrm{CHF}$ \\
\hline HTC Vive Cosmos & HTC & LCD & $110^{\circ}$ & $2880 \times 1700$ & $90 \mathrm{~Hz}$ & Inside-out & SteamVR & 2019 & $899 \mathrm{CHF}$ \\
\hline HTC Vive Pro & HTC & AMOLED & $110^{\circ}$ & $2880 \times 1600$ & $90 \mathrm{~Hz}$ & Outside-in & SteamVR & 2018 & $1249 \mathrm{CHF}$ \\
\hline Lenovo Explorer & Lenovo & LCD & $110^{\circ}$ & $2880 \times 1440$ & $90 \mathrm{~Hz}$ & Inside-out & WMR & 2018 & $489 \mathrm{CHF}$ \\
\hline Oculus Rift & Oculus & OLED & $110^{\circ}$ & $2160 \times 1200$ & $90 \mathrm{~Hz}$ & Outside-in & Oculus & 2016 & 399 \$ \\
\hline Oculus Rift S & Oculus & LCD & $115^{\circ}$ & $2560 \times 1440$ & $80 \mathrm{~Hz}$ & Inside-out & Oculus & 2019 & $530 \mathrm{CHF}$ \\
\hline Pimax 5K + & Pimax & LCD & $170^{\circ}$ & $5120 \times 1440$ & $144 \mathrm{~Hz}$ & Outside-in & SteamVR & 2018 & $928 \mathrm{CHF}$ \\
\hline Pimax 8K & Pimax & LCD & $170^{\circ}$ & $7680 \times 2160$ & $80 \mathrm{~Hz}$ & Outside-in & SteamVR & 2019 & $1099 \mathrm{CHF}$ \\
\hline Pimax 8K X & Pimax & LCD & $200^{\circ}$ & $7680 \times 2160$ & $80 \mathrm{~Hz}$ & Outside-in & SteamVR & 2020 & $1500 \mathrm{CHF}$ \\
\hline Playstation VR & Sony & OLED & $100^{\circ}$ & $1920 \times 1080$ & $120 \mathrm{~Hz}$ & Outside-in & PS4 & 2016 & $299 \mathrm{CHF}$ \\
\hline Samsung Odyssey+ & Samsung & AMOLED & $110^{\circ}$ & $3200 \times 1440$ & $90 \mathrm{~Hz}$ & Inside-out & WMR & 2017 & $538 \mathrm{CHF}$ \\
\hline StarVR One & StarVR & AMOLED & $210^{\circ}$ & $3660 \times 1464$ & $90 \mathrm{~Hz}$ & Outside-in & SteamVR & 2018 & $3199 \$$ \\
\hline Valve Index & Valve & LCD & $130^{\circ}$ & $2880 \times 1600$ & $144 \mathrm{~Hz}$ & Outside-in & SteamVR & 2019 & $1599 \mathrm{CHF}$ \\
\hline Varjo VR-1 & Varjo & AMOLED & $87^{\circ}$ & $1920 \times 1080$ & $90 \mathrm{~Hz}$ & Eyetracking & SteamVR & 2019 & $5999 \$$ \\
\hline XTAL & Vrgineers & OLED & $170^{\circ}$ & $5120 \times 1440$ & $70 \mathrm{~Hz}$ & Outside-in & SteamVR & 2018 & $5800 \$$ \\
\hline
\end{tabular}




\section{A. Appendix}

Table A.2.: Comparison of current VR headsets - Standalone.

\begin{tabular}{|l|l|l|l|l|l|l|l|l|l|}
\hline Name & Producer & Display & FOV & Resolution & Rate & Tracking & Platform & Date & Price \\
\hline HTC Vive Focus+ & HTC & AMOLED & $110^{\circ}$ & $2880 \times 1600$ & $75 \mathrm{~Hz}$ & Inside-out & Vive Wave & 2019 & $799 \$$ \\
\hline Mirage Solo & Lenovo & LCD & $110^{\circ}$ & $2560 \times 1440$ & $75 \mathrm{~Hz}$ & Inside-out & Daydream & 2018 & $425 \mathrm{CHF}$ \\
\hline Oculus Go & Oculus & LCD & $100^{\circ}$ & $2560 \times 1440$ & $72 \mathrm{~Hz}$ & 3 DOF & Oculus Mobile & 2018 & $219 \mathrm{CHF}$ \\
\hline Oculus Quest & Oculus & OLED & $100^{\circ}$ & $3200 \times 1440$ & $72 \mathrm{~Hz}$ & Inside-out & Oculus Mobile & 2019 & $479 \mathrm{CHF}$ \\
\hline Pico VR Goblin 2 & Pico & LCD & $90^{\circ}$ & $2880 \times 1600$ & $90 \mathrm{~Hz}$ & 3 DOF & - & 2017 & $399 \$$ \\
\hline
\end{tabular}

Table A.3.: Comparison of current VR headsets - Cardboard.

\begin{tabular}{|c|c|c|c|c|c|c|c|c|c|}
\hline Name & Producer & Display & $F O V$ & Resolution & Rate & Tracking & Platform & Date & Price \\
\hline Google Cardboard & Google & $* * *$ & $90^{\circ}$ & $* * *$ & $* * *$ & $3 \mathrm{DOF}$ & $* * *$ & 2014 & $12 \mathrm{CHF}$ \\
\hline Google Daydream View 2 & Google & $* * *$ & $100^{\circ}$ & $* * *$ & $* * *$ & $3 \mathrm{DOF}$ & Daydream & 2017 & $99 \$$ \\
\hline Merge & Merge & $* * *$ & $96^{\circ}$ & $* * *$ & $* * *$ & $3 \mathrm{DOF}$ & $* * *$ & 2017 & $49 \mathrm{CHF}$ \\
\hline Samsung Gear VR & Samsung & $* * *$ & $96^{\circ}$ & $* * *$ & $* * *$ & $3 \mathrm{DOF}$ & Oculus Mobile & 2017 & $99 \mathrm{CHF}$ \\
\hline VeeR Oasis VR & VeeR & $* * *$ & $112^{\circ}$ & $* * *$ & $* * *$ & $3 \mathrm{DOF}$ & $* * *$ & 2018 & $29 \$$ \\
\hline VR Shinecon & Shinecon & $* * *$ & $90^{\circ}$ & $* * *$ & $* * *$ & $3 \mathrm{DOF}$ & $* * *$ & 2018 & $43 \mathrm{CHF}$ \\
\hline Zeiss VR One+ & Zeiss & $* * *$ & $100^{\circ}$ & $* * *$ & $* * *$ & $3 \mathrm{DOF}$ & $* * *$ & 2015 & $109 \mathrm{CHF}$ \\
\hline
\end{tabular}

Table A.4.: PC requirements for tethered VR headsets.

\begin{tabular}{|c|c|c|c|c|c|c|}
\hline Name & Graphic Card & Processor & Memory & $O S$ & Video output & USB Ports \\
\hline HP Reverb & Nvidia GTX 1080 & Intel Core i7 & $16 \mathrm{~GB}$ & Windows 10 & $1 \mathrm{x}$ DisplayPort 1.3 & $1 \times$ USB 3.0 \\
\hline HTC Vive & Nvidia GTX 1070 & Intel Core i5-4590 & $4 \mathrm{~GB}$ & Windows 7 & 1 x DisplayPort 1.2 & $1 \times$ USB 2.0 \\
\hline HTC Vive Cosmos & Nvidia GTX 1070 & Intel Core i5-4590 & $8 \mathrm{~GB}$ & Windows 10 & 1 x DisplayPort 1.2 & $1 \times$ USB 3.0 \\
\hline HTC Vive Pro & Nvidia GTX 1070 & Intel Core i5-4590 & $4 \mathrm{~GB}$ & Windows 10 & 1 x DisplayPort 1.2 & $1 \times$ USB 3.0 \\
\hline Lenovo Explorer & Nvidia GTX 1060 & Intel Core i5-4590 & $8 \mathrm{~GB}$ & Windows 10 & 1 x DisplayPort 1.2 & $1 \times$ USB 3.0 \\
\hline Oculus Rift & Nvidia GTX 1060 & Intel Core i5-4590 & $8 \mathrm{~GB}$ & Windows 10 & 1 x HDMI 1.3 & $3 \times$ USB 3.0 \\
\hline Oculus Rift S & Nvidia GTX 1060 & Intel Core i5-4590 & $8 \mathrm{~GB}$ & Windows 10 & 1 x DisplayPort 1.2 & $1 \times$ USB 3.0 \\
\hline Oculus Quest + Link & Nvidia GTX 1080 & Intel Core i5-4590 & $8 \mathrm{~GB}$ & Windows 10 & - & $1 \times$ USB 3.0 \\
\hline Pimax 5K, 5K+ & Nvidia GTX 1070 & Intel Core i5-4590 & $8 \mathrm{~GB}$ & Windows $8.1,10$ & 1 x DisplayPort 1.4 & $1 \times$ USB 3.0 \\
\hline Pimax 8K+ & Nvidia GTX $1080 \mathrm{Ti}$ & Intel Core i5-9400 & $8 \mathrm{~GB}$ & Windows 10 & 1 x DisplayPort 1.2 & $1 \times$ USB 3.0 \\
\hline Pimax 8K X & Nvidia RTX 2060 & Intel Core i5-9400 & $8 \mathrm{~GB}$ & Windows 10 & 1 x DisplayPort 1.2 & $1 \times$ USB 3.0 \\
\hline Samsung Odyssey+ & Nvidia GTX 1060 & Intel Core i5-4590 & $8 \mathrm{~GB}$ & Windows 10 & 1 x DisplayPort 1.2 & $1 \times$ USB 3.0 \\
\hline StarVR & Nvidia GTX $1080 \mathrm{Ti}$ & Intel Core i7-7700 & $16 \mathrm{~GB}$ & Windows 10 & - & - \\
\hline Valve Index & Nvidia GTX 1070 & Quad Core0 & $8 \mathrm{~GB}$ & Windows 10 & 1 x DisplayPort 1.2 & $1 \times$ USB 3.0 \\
\hline Varjo VR-2 & Nvidia RTX 2080 & Intel Core i7-6700 & $32 \mathrm{~GB}$ & Windows 10 & 2 x DisplayPort 1.2 & $1 \times$ USB 3.0 \\
\hline
\end{tabular}


Table A.5.: Data set of the SUS questionnaire - Part 1.

\begin{tabular}{|c|c|c|c|c|c|c|c|c|c|c|c|c|c|c|}
\hline \# & Gender & Profession & $A p p$ & $Q 1$ & $Q^{2}$ & $Q 3$ & $Q 4$ & $Q 5$ & Q6 & $Q 7$ & $Q 8$ & $Q^{9}$ & $Q 10$ & SUS \\
\hline 1 & Male & Medical & VR-side & 4 & 2 & 4 & 2 & 5 & 1 & 5 & 1 & 4 & 2 & 85.0 \\
\hline 1 & Male & Medical & Control & 5 & 1 & 4 & 2 & 4 & 1 & 4 & 1 & 5 & 3 & 85.0 \\
\hline 2 & Female & Medical & VR-side & 4 & 2 & 5 & 2 & 4 & 1 & 5 & 1 & 4 & 1 & 87.5 \\
\hline 2 & Female & Medical & Control & 4 & 2 & 4 & 3 & 4 & 2 & 4 & 1 & 4 & 2 & 75.0 \\
\hline 3 & Male & Nurse & VR-side & 4 & 1 & 5 & 1 & 5 & 1 & 5 & 1 & 5 & 1 & 97.5 \\
\hline 3 & Male & Nurse & Control & 5 & 1 & 5 & 1 & 4 & 1 & 4 & 1 & 5 & 2 & 92.5 \\
\hline 4 & Female & Therapist & VR-side & 5 & 1 & 5 & 4 & 5 & 1 & 5 & 1 & 5 & 2 & 90.0 \\
\hline 4 & Female & Therapist & Control & 5 & 1 & 5 & 5 & 5 & 1 & 4 & 1 & 4 & 4 & 77.5 \\
\hline 5 & Female & Psychologist & VR-side & 4 & 3 & 3 & 3 & 4 & 1 & 3 & 2 & 3 & 3 & 62.5 \\
\hline 5 & Female & Psychologist & Control & 4 & 3 & 2 & 3 & 4 & 1 & 3 & 4 & 3 & 4 & 52.5 \\
\hline 6 & Female & Psychologist & VR-side & 3 & 1 & 5 & 1 & 4 & 2 & 5 & 1 & 5 & 1 & 90.0 \\
\hline 6 & Female & Psychologist & Control & 4 & 1 & 5 & 1 & 4 & 2 & 4 & 1 & 5 & 1 & 90.0 \\
\hline 7 & Male & Medical & VR-side & 5 & 1 & 5 & 1 & 5 & 1 & 5 & 1 & 5 & 1 & 100 \\
\hline 7 & Male & Medical & Control & 5 & 1 & 5 & 5 & 5 & 1 & 4 & 1 & 4 & 1 & 85.0 \\
\hline 8 & Female & Medical & VR-side & 5 & 2 & 4 & 3 & 5 & 1 & 5 & 1 & 5 & 1 & 90.0 \\
\hline 8 & Female & Medical & Control & 5 & 1 & 4 & 3 & 5 & 1 & 5 & 1 & 4 & 1 & 90.0 \\
\hline 9 & Male & Nurse & VR-side & 3 & 1 & 5 & 3 & 3 & 2 & 4 & 2 & 4 & 2 & 72.5 \\
\hline 9 & Male & Nurse & Control & 4 & 2 & 4 & 3 & 4 & 2 & 4 & 2 & 4 & 1 & 75.0 \\
\hline 10 & Female & Nurse & VR-side & 4 & 1 & 4 & 5 & 4 & 2 & 4 & 1 & 3 & 3 & 67.5 \\
\hline 10 & Female & Nurse & Control & 2 & 1 & 5 & 2 & 4 & 2 & 5 & 1 & 4 & 4 & 75.0 \\
\hline 11 & Female & Nurse & VR-side & 5 & 1 & 5 & 2 & 4 & 2 & 5 & 1 & 5 & 1 & 92.5 \\
\hline 11 & Female & Nurse & Control & 4 & 1 & 5 & 2 & 4 & 2 & 5 & 1 & 4 & 1 & 87.5 \\
\hline 12 & Female & Nurse & VR-side & 4 & 2 & 3 & 4 & 4 & 2 & 3 & 2 & 1 & 3 & 55.0 \\
\hline 12 & Female & Nurse & Control & 5 & 2 & 4 & 3 & 4 & 2 & 4 & 2 & 3 & 2 & 72.5 \\
\hline 13 & Female & Administ. & VR-side & 3 & 2 & 3 & 4 & 4 & 3 & 3 & 2 & 3 & 2 & 57.5 \\
\hline 13 & Female & Administ. & Control & 5 & 2 & 4 & 2 & 4 & 2 & 4 & 1 & 3 & 2 & 77.5 \\
\hline 14 & Female & Administ. & VR-side & 2 & 1 & 5 & 2 & 5 & 2 & 5 & 1 & 5 & 1 & 87.5 \\
\hline 14 & Female & Administ. & Control & 2 & 2 & 5 & 3 & 5 & 2 & 4 & 1 & 5 & 1 & 80.0 \\
\hline 15 & Female & Administ. & VR-side & 2 & 2 & 5 & 5 & 4 & 3 & 3 & 2 & 4 & 2 & 60.0 \\
\hline 15 & Female & Administ. & Control & 4 & 2 & 4 & 4 & 3 & 3 & 4 & 2 & 4 & 2 & 65.0 \\
\hline 16 & Female & Nurse & VR-side & 5 & 1 & 5 & 4 & 5 & 1 & 5 & 1 & 4 & 4 & 82.5 \\
\hline 16 & Female & Nurse & Control & 5 & 1 & 4 & 4 & 5 & 1 & 5 & 1 & 5 & 4 & 82.5 \\
\hline 17 & Female & Nurse & VR-side & 5 & 1 & 5 & 2 & 4 & 2 & 4 & 1 & 4 & 1 & 87.5 \\
\hline 17 & Female & Nurse & Control & 4 & 2 & 4 & 2 & 4 & 2 & 4 & 2 & 4 & 2 & 75.0 \\
\hline 18 & Female & Psychologist & VR-side & 4 & 2 & 4 & 2 & 4 & 1 & 4 & 2 & 4 & 2 & 77.5 \\
\hline 18 & Female & Psychologist & Control & 4 & 1 & 4 & 1 & 4 & 1 & 4 & 2 & 4 & 1 & 85.0 \\
\hline 19 & Male & Nurse & VR-side & 4 & 1 & 4 & 3 & 5 & 2 & 4 & 1 & 4 & 1 & 82.5 \\
\hline 19 & Male & Nurse & Control & 5 & 1 & 4 & 3 & 4 & 1 & 4 & 2 & 4 & 2 & 80.0 \\
\hline
\end{tabular}




\section{A. Appendix}

Table A.6.: Data set of the SUS questionnaire - Part 2.

\begin{tabular}{|c|c|c|c|c|c|c|c|c|c|c|c|c|c|c|}
\hline$\#$ & Gender & Profession & App & $Q 1$ & $Q 2$ & Q3 & $Q 4$ & $Q 5$ & $Q 6$ & $Q 7$ & $Q 8$ & $Q 9$ & $Q 10$ & SUS \\
\hline 20 & Female & Therapist & VR-side & 4 & 2 & 5 & 1 & 5 & 2 & 3 & 1 & 2 & 1 & 80.0 \\
\hline 20 & Female & Therapist & Control & 4 & 2 & 4 & 2 & 4 & 3 & 4 & 1 & 3 & 2 & 72.5 \\
\hline 21 & Male & Nurse & VR-side & 3 & 2 & 3 & 3 & 3 & 3 & 2 & 2 & 3 & 3 & 52.5 \\
\hline 21 & Male & Nurse & Control & 3 & 2 & 3 & 2 & 3 & 3 & 2 & 3 & 2 & 3 & 50.0 \\
\hline 22 & Male & Medical & VR-side & 4 & 1 & 4 & 2 & 5 & 2 & 5 & 1 & 5 & 1 & 90.0 \\
\hline 22 & Male & Medical & Control & 5 & 1 & 5 & 2 & 5 & 1 & 5 & 1 & 5 & 1 & 97.5 \\
\hline 23 & Male & Nurse & VR-side & 3 & 2 & 2 & 5 & 4 & 3 & 3 & 1 & 2 & 2 & 52.5 \\
\hline 23 & Male & Nurse & Control & 4 & 1 & 3 & 4 & 3 & 2 & 2 & 4 & 2 & 4 & 47.5 \\
\hline 24 & Female & Nurse & VR-side & 5 & 1 & 5 & 4 & 5 & 1 & 4 & 1 & 3 & 1 & 85.0 \\
\hline 24 & Female & Nurse & Control & 5 & 1 & 5 & 4 & 4 & 1 & 5 & 1 & 4 & 1 & 87.5 \\
\hline 25 & Female & Administ. & VR-side & 5 & 2 & 4 & 2 & 5 & 2 & 4 & 2 & 5 & 1 & 85.0 \\
\hline 25 & Female & Administ. & Control & 5 & 2 & 5 & 2 & 4 & 2 & 4 & 1 & 2 & 1 & 80.0 \\
\hline 26 & Female & Administ. & VR-side & 3 & 1 & 5 & 2 & 5 & 2 & 5 & 1 & 5 & 1 & 90.0 \\
\hline 26 & Female & Administ. & Control & 5 & 1 & 5 & 1 & 4 & 2 & 5 & 1 & 5 & 2 & 92.5 \\
\hline 27 & Female & Administ. & VR-side & 2 & 2 & 4 & 2 & 4 & 2 & 4 & 2 & 4 & 1 & 72.5 \\
\hline 27 & Female & Administ. & Control & 3 & 2 & 4 & 2 & 4 & 2 & 4 & 2 & 3 & 2 & 70.0 \\
\hline 28 & Female & Nurse & VR-side & 5 & 1 & 5 & 1 & 5 & 1 & 5 & 1 & 4 & 1 & 97.5 \\
\hline 28 & Female & Nurse & Control & 5 & 1 & 5 & 1 & 5 & 1 & 5 & 1 & 5 & 1 & 100 \\
\hline 29 & Female & Medical & VR-side & 5 & 1 & 5 & 2 & 5 & 1 & 5 & 1 & 5 & 1 & 97.5 \\
\hline 29 & Female & Medical & Control & 5 & 1 & 5 & 1 & 5 & 1 & 4 & 1 & 5 & 1 & 97.5 \\
\hline 30 & Male & Medical & VR-side & 4 & 1 & 5 & 1 & 4 & 2 & 3 & 1 & 3 & 2 & 80.0 \\
\hline 30 & Male & Medical & Control & 5 & 1 & 5 & 1 & 4 & 1 & 3 & 1 & 5 & 1 & 92.5 \\
\hline 31 & Female & Nurse & VR-side & 5 & 1 & 5 & 5 & 5 & 1 & 5 & 1 & 5 & 1 & 90.0 \\
\hline 31 & Female & Nurse & Control & 5 & 1 & 4 & 5 & 5 & 2 & 4 & 2 & 3 & 4 & 67.5 \\
\hline 32 & Male & Medical & VR-side & 4 & 1 & 5 & 1 & 5 & 1 & 4 & 1 & 4 & 1 & 92.5 \\
\hline 32 & Male & Medical & Control & 5 & 1 & 4 & 1 & 4 & 1 & 5 & 1 & 4 & 2 & 90.0 \\
\hline 33 & Female & Nurse & VR-side & 3 & 1 & 5 & 2 & 3 & 1 & 4 & 2 & 1 & 2 & 70.0 \\
\hline 33 & Female & Nurse & Control & 4 & 2 & 4 & 3 & 4 & 2 & 4 & 2 & 2 & 3 & 65.0 \\
\hline 34 & Female & Other & VR-side & 4 & 2 & 4 & 5 & 4 & 2 & 4 & 2 & 3 & 2 & 65.0 \\
\hline 34 & Female & Other & Control & 4 & 2 & 3 & 4 & 4 & 2 & 4 & 2 & 3 & 3 & 62.5 \\
\hline 35 & Female & Nurse & VR-side & 4 & 1 & 5 & 1 & 4 & 3 & 5 & 4 & 5 & 3 & 77.5 \\
\hline 35 & Female & Nurse & Control & 3 & 2 & 5 & 1 & 5 & 3 & 5 & 3 & 4 & 3 & 75.0 \\
\hline 36 & Female & Nurse & VR-side & 4 & 1 & 5 & 3 & 4 & 4 & 4 & 1 & 5 & 1 & 80.0 \\
\hline 36 & Female & Nurse & Control & 4 & 1 & 5 & 2 & 5 & 2 & 5 & 1 & 5 & 2 & 90.0 \\
\hline 37 & Female & Nurse & VR-side & 4 & 2 & 4 & 3 & 4 & 3 & 4 & 1 & 3 & 3 & 67.5 \\
\hline 37 & Female & Nurse & Control & 4 & 2 & 4 & 3 & 4 & 3 & 4 & 2 & 4 & 3 & 67.5 \\
\hline 38 & Female & Psychologist & VR-side & 5 & 1 & 4 & 3 & 3 & 3 & 4 & 2 & 5 & 2 & 75.0 \\
\hline 38 & Female & Psychologist & Control & 4 & 3 & 3 & 4 & 4 & 3 & 4 & 3 & 4 & 2 & 60.0 \\
\hline
\end{tabular}




\section{Bibliography}

[1] 3D assets, Interior Living Room. https://assetstore.unity.com/ packages/3d/environments/bfw-archviz-interior-living-room93941. (accessed 20.09.2020).

[2] HP Reverb VR Headset. https://store.hp.com/SwitzerlandStore/ Merch/Offer.aspx?p=b-hp-reverb-vr-headset \&lang=en-CH. (accessed 20.09.2020).

[3] Image of the HP Reverb VR Headset. https: //www . fotointern.ch/archiv/ 2019/03/25/hp-bringt-hochaufloesendes-vr-headset-hp-reverbin-die-schweiz/. (accessed 20.09.2020).

[4] Image of the Medion Erazer X87092. https://www.digitec.ch/en/s1/ product/medion-erazer-x87092-intel-core-i7-9700-16gb-512gbssd-pc-12435156\#gallery-open. (accessed 20.09.2020).

[5] Image of the Roland VT-4 Voice Transformer. https://static.roland.com/ products/vt-4/images/hero.png. (accessed 20.09.2020).

[6] Inside-Out tracking vs. Outside-In tracking. https://arvrtips.com/insideout-outside-in-vr-tracking/. (accessed 20.09.2020).

[7] Medion Erazer X87092. https://www.digitec.ch/en/s1/product/ medion-erazer-x87092-intel-core-i7-9700-16gb-512gb-ssd-pc12435156 . (accessed 20.09.2020).

[8] Microsoft Visual Studio. https://docs.microsoft.com/en-us/ visualstudio/releasenotes/vs2017-relnotes. (accessed 20.09.2020).

[9] Mixamo, Animate 3D Characaters. https://www.mixamo.com/\#/. (accessed 20.09.2020). 
[10] Mixed Reality Portal. https://www.microsoft.com/en-us/p/mixedreality-portal/9ng1h8b3zc7m?activetab=pivot:overviewtab. (accessed 20.09.2020).

[11] Roland VT-4 Voice Transformer. https://www.roland.com/dech/products/vt-4/. (accessed 20.09.2020).

[12] SALSA, Real time LipSync asset for Unity. https: / / crazyminnowstudio.com/ unity-3d/lip-sync-salsa/. (accessed 20.09.2020).

[13] Screen-door effect. https://whatis.techtarget.com/definition/ screen-door-effect. (accessed 20.09.2020).

[14] Setting Up HP VR Reverb Headset. https://support.hp.com/in-en/ document/c06433655. (accessed 20.09.2020).

[15] System Usability Scale. https: / /www.usability.gov/how-to-and-tools / methods/system-usability-scale.html. (accessed 20.09.2020).

[16] UMA - Unity Multipurpose Avatar. https://assetstore.unity.com/ packages/3d/characters/uma-2-unity-multipurpose-avatar35611. (accessed 20.09.2020).

[17] UMA Clothing Pack. https://assetstore.unity.com/packages/3d/ characters/uma-clothing-pack-119640. (accessed 20.09.2020).

[18] UMA Face Pack. https://assetstore.unity.com/packages/3d/ characters/uma-face-pack-vol-1-40959. (accessed 20.09.2020).

[19] UMA Hair Pack. https://assetstore.unity.com/packages/3d/ characters/uma-hair-pack-1-26521. (accessed 20.09.2020).

[20] Understanding VR HMD Display Technology. https: / / realnewworld.com/vrhmd-display-technology/. (accessed 20.09.2020).

[21] Unity, 3D development platform. https: / / unity. com/. (accessed 20.09.2020).

[22] Unity Asset Store. https://assetstore.unity. com/. (accessed 20.09.2020).

[23] Aaron Bangor, Philip T Kortum, and James T Miller. An empirical evaluation of the system usability scale. Intl. Journal of Human-Computer Interaction, 24(6):574-594, 2008.

[24] Nahila Bonfiglio. Deepmind partners with gaming company for ai research. The Daily Dot, 2018.

[25] Sue Bregman. Assertiveness training for mentally retarded adults. Mental Retardation, 1984.

[26] Tom KJ Craig, Mar Rus-Calafell, Thomas Ward, Julian P Leff, Mark Huckvale, Elizabeth Howarth, Richard Emsley, and Philippa A Garety. Avatar therapy for auditory verbal hallucinations in people with psychosis: a single-blind, randomised controlled trial. The Lancet Psychiatry, 5(1):31-40, 2018.

[27] Olivier Percie du Sert, Stéphane Potvin, Olivier Lipp, Laura Dellazizzo, Mélanie Lau- 
relli, Richard Breton, Pierre Lalonde, Kingsada Phraxayavong, Kieron O'Connor, JeanFrançois Pelletier, et al. Virtual reality therapy for refractory auditory verbal hallucinations in schizophrenia: a pilot clinical trial. Schizophrenia research, 197:176-181, 2018.

[28] Maryrose Gerardi, Judith Cukor, JoAnn Difede, Albert Rizzo, and Barbara Olasov Rothbaum. Virtual reality exposure therapy for post-traumatic stress disorder and other anxiety disorders. Current psychiatry reports, 12(4):298-305, 2010.

[29] Selen Turkay and Charles K Kinzer. The effects of avatar-based customization on player identification. International Journal of Gaming and Computer-Mediated Simulations (IJGCMS), 6(1):1-25, 2014. 

Master Thesis Project Description

Virtual Reality Avatar Therapy for People Hearing Voices

Mischa Brander

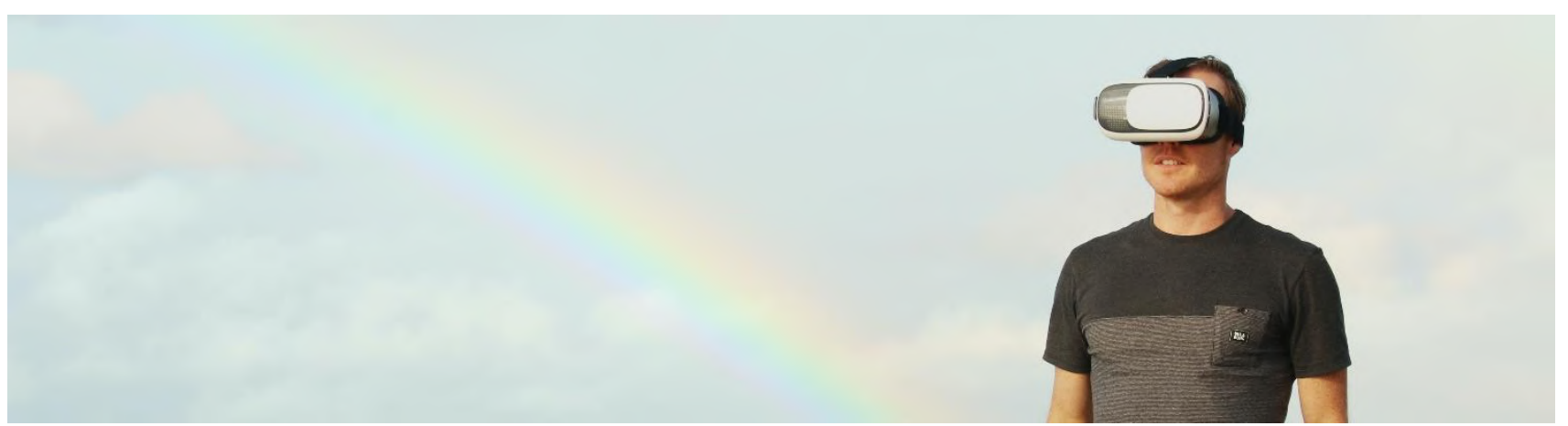

\section{Introduction}

Hearing voices is probably the worst form of acoustic hallucinations. It can be experienced as severely disturbing and is influenced by diverse factors including the ability of the individual to influence and control the hallucinatory experience itself. In recent years virtual reality (VR) has become a treatment option. In the so-called Avatar Therapy, patients with schizophrenia and acoustic hallucinations design a visual and auditory recreation (avatar) of the entity to which they attribute their hallucinations. The goal of this project is to help doctors at USZ, led by Dr. Stephan Egger, to develop an advanced VR Avatar Therapy, as described in the tasks below.

\section{Task Description}

Research. Discuss with medical personal and research existing literature on VR for agent therapy and schizophrenia rehabilitation. Hardware. Choose, acquire and setup the necessary hardware. Basic VR setup. Setup a VR app with a simple scene in which an avatar, controlled by the therapist, interacts with the patient who is wearing a VR headset. The app consists of two parts: a server running on a powerful computer and rendering the scene for the patient, and a remote client for the therapist, allowing the latter to control the avatar and speak through it using lip sync and a voice modification device. Avatar customisation. Allow the patient to customise the avatar. Advanced avatar movement. Allow the therapist to puppet the avatar in a natural way. Evaluation. Support the USZ team to test and evaluate the system with patients.

\section{Milestones}

\begin{tabular}{|l|l|}
\hline Task & Deadline \\
\hline Start & April 1 \\
\hline Discuss with medical personal and research existing literature & April 15 \\
\hline Hardware setup & April 29 \\
\hline Basic VR setup & May 20 \\
\hline Avatar customisation & June 17 \\
\hline Midterm presentation & TBA, late June \\
\hline Advanced avatar movement & July 15 \\
\hline Evaluation & September 2 \\
\hline Hand-in final thesis & October 1 \\
\hline Thesis presentation & TBA \\
\hline
\end{tabular}

\section{Remarks}

A written report and an oral presentation conclude the thesis. The thesis will be overseen by Prof. Robert W. Sumner and supervised by Dr. Stephan Egger and Dr. Stéphane Magnenat. 

Eidgenössische Technische Hochschule Zürich

Swiss Federal Institute of Technology Zurich

\section{Declaration of originality}

The signed declaration of originality is a component of every semester paper, Bachelor's thesis, Master's thesis and any other degree paper undertaken during the course of studies, including the respective electronic versions.

Lecturers may also require a declaration of originality for other written papers compiled for their courses.

I hereby confirm that I am the sole author of the written work here enclosed and that I have compiled it in my own words. Parts excepted are corrections of form and content by the supervisor.

Title of work (in block letters):

Virtual Reality Avatar Therapy for People Hearing Voices

Authored by (in block letters):

For papers written by groups the names of all authors are required.

Name(s):

First name(s):

Brander

Mischa

With my signature I confirm that

- I have committed none of the forms of plagiarism described in the 'Citation etiquette' information sheet.

- I have documented all methods, data and processes truthfully.

- I have not manipulated any data.

- I have mentioned all persons who were significant facilitators of the work.

I am aware that the work may be screened electronically for plagiarism.

Place, date

Signature(s)

Schlieren, 1.10.2020

For papers written by groups the names of all authors are required. Their signatures collectively guarantee the entire content of the written paper. 



\section{Vereinbarung über Urheberrechte an Computerprogrammen von Studierenden}

Arbeit:
Semesterarbeit
Bachelorarbeit
X Masterarbeit
Andere

Titel der Arbeit:

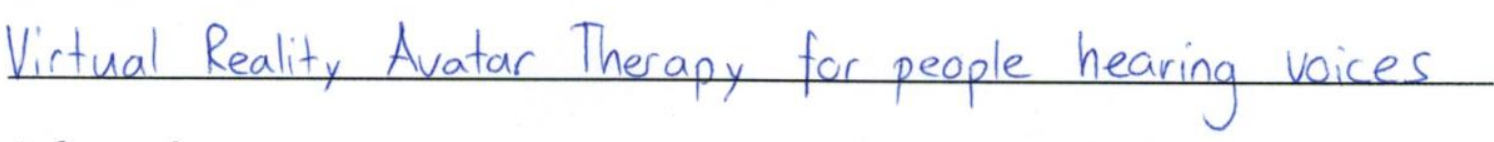

Auftraggeber:

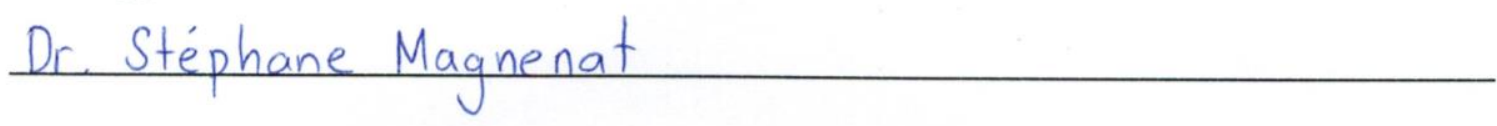

\section{Studierender:}

Name, Vorname:

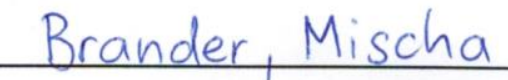
ETH-Nr: $14-918-064$

\section{Vereinbarung:}

\section{Gegenstand dieser Vereinbarung}

Regelung der Urheberrechte an Computerprogrammen, die im Rahmen der Ausbildung auf Grund einer Aufgabenstellung seitens der Hochschule durch Studierende ohne Bezahlung geschrieben werden, auf der Basis des Urheberrechtsgesetzes vom 9. Oktober 1992.

\section{Verwendungs- und Änderungsbefugnisse}

Computerprogramme aus der oben genannten Arbeit dürfen nach deren Abschluss sowohl seitens der Hochschule wie auch vom Studierenden beliebig und vergütungsfrei verwendet werden; sie dürfen auch verändert werden.

\section{Autorenhinweise}

Werden grössere Programmmodule weitgehend unverändert verwendet, soll auf den Studierenden sowie auf den Aufgabensteller in geeigneter Form hingewiesen werden.

\section{Urheberrechte an Texten}

Die Urheberrechte an den zugehörigen Berichten und Dokumentationen stehen dem Studierenden zu; die Hochschule kann diese für geeignete Zwecke und in Bibliotheken vergütungsfrei verwenden.

Zürich,

Auftraggeber: zürich, 02.04 .2020

Studierender:

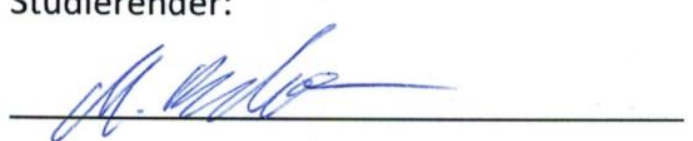


